ISS Interface Mechanisms and their Heritage

The International Space Station, by nurturing technological development of a variety of pressurized and unpressurized interface mechanisms fosters "competition at the technology level". Such redundancy and diversity allows for the development and testing of mechanisms that might be used for future exploration efforts. The International space Station, as a test-bed for exploration, has 4 types of pressurized interfaces between elements and 6 unpressurized attachment mechanisms. Lessons learned from the design, test and operations of these mechanisms will help inform the design for a new international standard pressurized docking mechanism for the NASA Docking System. This paper will examine the attachment mechanisms on the ISS and their attributes. It will also look ahead at the new NASA docking system and trace its lineage to heritage mechanisms. 


\title{
ISS Interface Mechanisms and their Heritage
}

\author{
John Cook ${ }^{1}$, Valery Aksamentov ${ }^{2}$, Thomas Hoffman ${ }^{3}$, \\ and \\ Wes Bruner ${ }^{4}$ \\ The Boeing Company, 13100 Space Center Boulevard, Houston, Texas, 77059
}

The International Space Station, by requiring technological development of a variety of pressurized and unpressurized interface mechanisms fosters "competition at the technology level". Such redundancy and diversity allows for the development and testing of mechanisms that might be used for future exploration efforts. The International Space Station, as a test-bed for exploration, has four types of pressurized interfaces between elements and nine unpressurized attachment mechanisms. Lessons learned from the design, test and operations of these mechanisms will aid in the design for a new International Standard pressurized docking mechanism for future NASA and commercial vehicles. This paper will examine the attachment mechanisms on the ISS and their attributes. It will also look ahead at the new NASA Docking System and trace its lineage to heritage mechanisms.

\section{Nomenclature}

$\begin{array}{ll}\text { ACBM } & =\text { Active Common Berthing Mechanism } \\ \text { AMS } & =\text { Alpha Magnetic Spectrometer } \\ \text { APAS } & =\text { Androgynous Peripheral Attachment System } \\ C A S & =\text { Carrier Attachment System } \\ C B M & =\text { Common Berthing Mechanism } \\ C S M & =\text { Command Service Module } \\ E F & =\text { Exposed Facility } \\ E F & =\text { Exposed Facility Unit } \\ E F B M & =\text { Exposed Facility Berthing Mechanism } \\ E L C & =\text { Express Logistics Carrier } \\ E S P 3 & =\text { External Stowage Platform Three }\end{array}$

\footnotetext{
${ }^{1}$ Mechanical System Design \& Analysis Engineer 3, EVA \& On Orbit Support, The Boeing Company, 13100 Space Center Boulevard, HB6-20, Houston, Texas, 77059

${ }^{2}$ ISS Life Extension Project Manager, The Boeing Company, 13100 Space Center Boulevard, HB6-40, Houston, Texas, 77059.

${ }^{3}$ Subsystem Manager, Orbiter Docking System, The Boeing Company, 13100 Space Center Boulevard, HB6-40, Houston, Texas, 77059.

${ }^{4}$ Structural Analysis Engineer 5, Advanced Integrated Analysis, The Boeing Company, 13100 Space Center Boulevard, HB2-10, Houston, Texas, 77059.
} 


\begin{tabular}{|c|c|}
\hline ES & $=$ Exposed Segment \\
\hline$F G B$ & = Functional Cargo Block [sic] (Functionalui Germaticheskii Block) \\
\hline ISS & $=$ International Space Station \\
\hline$J E M$ & = Japanese Experiment Module \\
\hline$L M$ & $=$ Lunar Module \\
\hline MBS & $=$ Mobile Remote Servicer Base System \\
\hline MRTAS & = Modified Rocketdyne Truss Attachment Mechanism \\
\hline MTSAS & $=$ Module to Truss Segment Attachment System \\
\hline MT & = Mobile Transporter \\
\hline$N E O$ & $=$ Near Earth Object \\
\hline$P 1$ & $=$ Port One \\
\hline$P 3$ & $=$ Port Three \\
\hline P6 & $=$ Port Six \\
\hline PCBM & = Passive Common Berthing Mechanism \\
\hline$P M A$ & $=$ Pressurized Mating Adapter \\
\hline$P M$ & $=$ Pressurized Module \\
\hline$R T L$ & = Ready To Latch \\
\hline RTAS & = Rocketdyne Truss Attachment System \\
\hline SO & $=$ Starboard Zero \\
\hline S1 & = Starboard One \\
\hline S3 & = Starboard Three \\
\hline SSAS & = Segment to Segment Attachment System \\
\hline US & $=$ United States \\
\hline USSR & $=$ Union of Soviet Socialist Republics \\
\hline$Z 1$ & = Zenith One \\
\hline
\end{tabular}




\section{Introduction}

${ }^{6} \mathbf{B}^{\text {efore man can truly call himself the master of space, he must master orbital rendezvous. This technique may }}$ ultimately make it possible to assemble the huge space stations and the massive interplanetary craft required for manned expeditions to Venus, Mars, and perhaps as far as Pluto on the outer rim of the solar system, much sooner than if direct flight were necessary. Rendezvous may also enable astronauts of the future to refuel, repair, and resupply spacecraft, rescue other astronauts from disabled craft, and ferry crews between the earth and space stations or between space platforms and planets.” [1] Since these thoughts were expressed in the early 1960s, there has been much development of mechanisms and systems to perform the critical operation of joining vehicles in space. The primary means of mating spacecraft are docking and berthing.

\section{Definition of Docking and Berthing}

Docking is when one incoming spacecraft rendezvous with another spacecraft and flies a controlled collision trajectory in such a manner so as to align and mesh the interface mechanisms. The spacecraft docking mechanisms typically enter what is called soft capture, followed by a load attenuation phase, and then the hard docked position which establishes an air-tight structural connection between spacecraft.

Berthing, by contrast, is when an incoming spacecraft is grappled by a robotic arm and its interface mechanism is placed in close proximity of the stationary interface mechanism. Then typically there is a capture process, coarse alignment and fine alignment and then structural attachment. This will be discussed in more detail in later chapters. A family tree of docking and berthing mechanisms is shown in Figure 1. It shows the chronological development of mating mechanisms from the world's space faring nations. 
Family Tree of Docking and Berthing Mechanisms

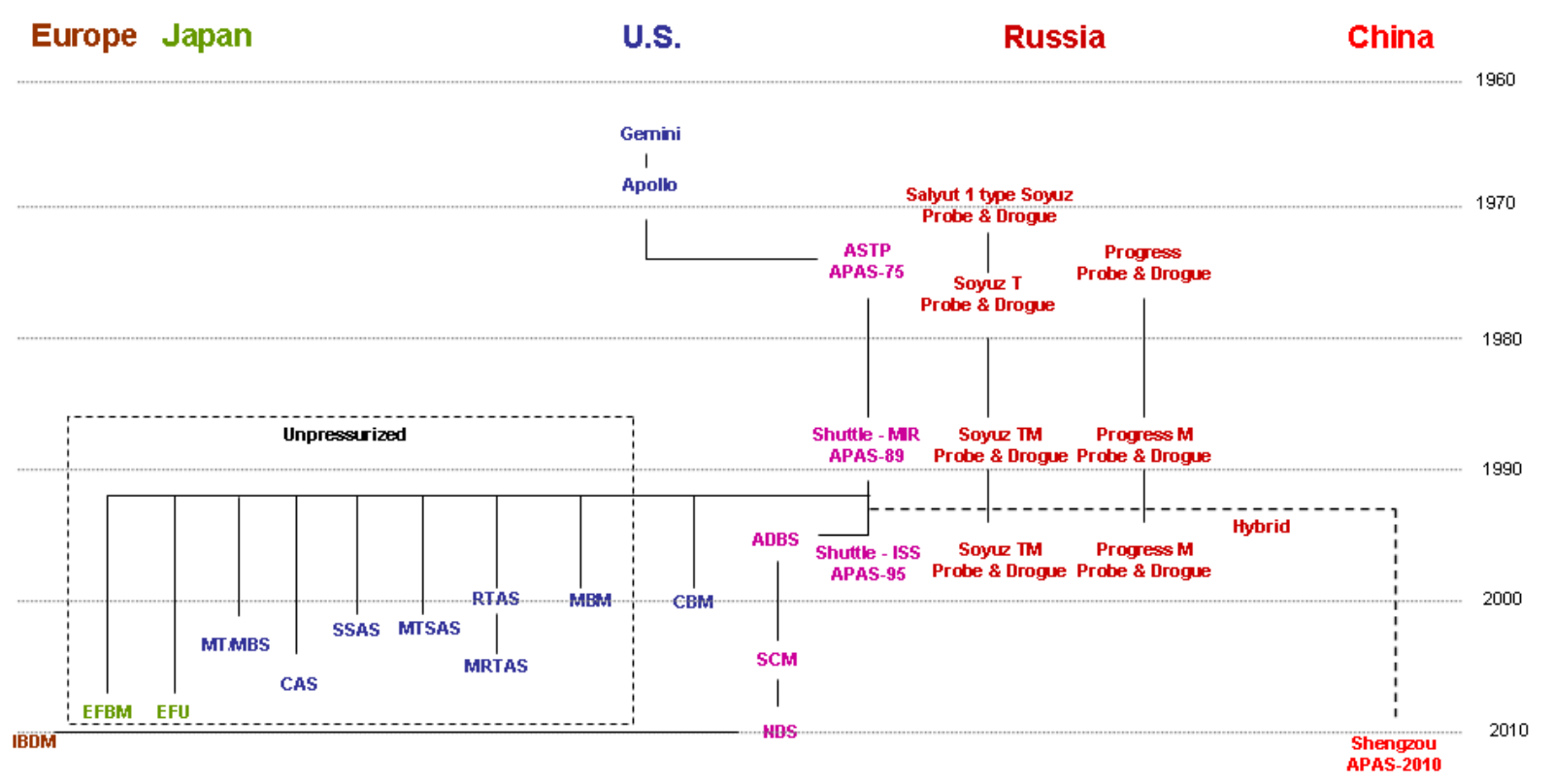

Fig. 1 A conceptual family tree of Docking and Berthing Mechanisms 


\section{History of Docking and Berthing}

\section{A. Gemini}

The Russians were the first into space, but the Americans, using a spacecraft developed by Boeing heritage McDonnell Aircraft were the first to dock, during the Gemini Program. Based on developments since Gemini, The Boeing Company is uniquely qualified in the role of on-orbit rendezvous and docking and berthing. As illustrated in Figure 1, our experience and history make us the leader in Human Spaceflight docking and berthing. The main Gemini program objectives were to demonstrate the ability to spend longer periods in space than were experienced in the Mercury program, change orbital planes and to rendezvous and dock in space. Gemini VIII, on March 16, 1966 was the first mission to dock. The McDonnell Aircraft interface mechanism played a key role in that. It used a relatively simple cone and cup interface, shown in Figure 2. 


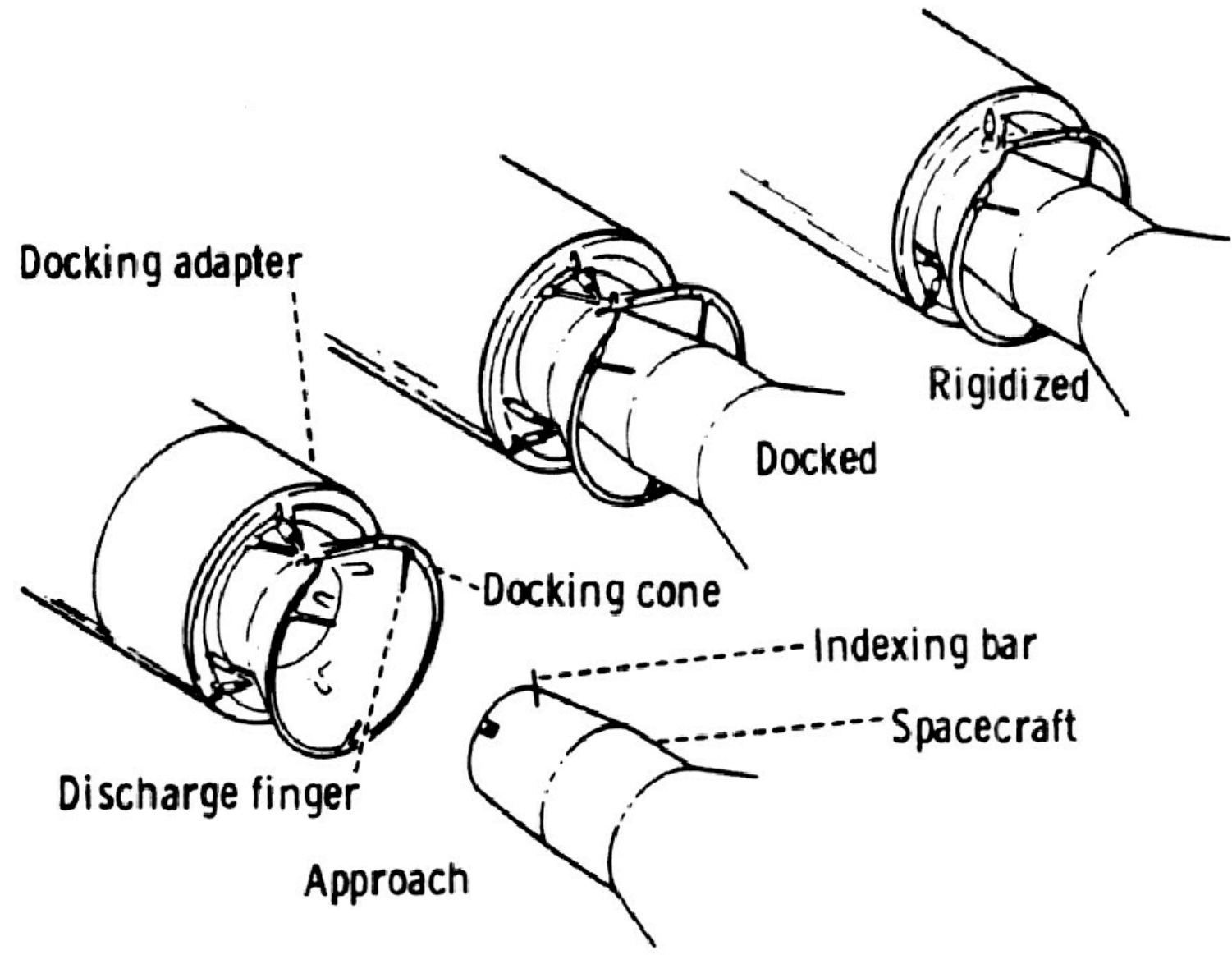

Fig. 2 Overview of Gemini Docking Mechanism

Image courtesy of NASA [2]

This interface is characterized by the male portion of the interface on the Gemini spacecraft which seats in the female portion on the Agena spacecraft. This cone has an alignment feature, called an indexing bar, which interacted with a V-shaped notch in the Agena vehicle. There was only one relative Gemini/Agena orientation that Gemini docking could occur. The Gemini program successfully demonstrated that two spacecraft could rendezvous and dock on orbit. The mechanism did not allow crew to transfer between modules.

"When the distance between vehicles was 250 miles radar was switched on. As the gap closed to 50 miles, the Gemini astronauts picked up the Agena's flashing beacon and took over control of Gemini. Aiding the astronauts was the status display panel outwardly mounted on the Agena-D, giving visual data on Agena fuel reserves, electrical power, and attitude position. During rendezvous maneuvers the relative speed between the vehicles was cut to less than $2 \mathrm{mph}$, so that when docking, their noses touched gently. On contact, the Gemini's narrow end 
entered the Agena's target docking adapter, whose latches clamped shut to prevent the two vehicles from slipping apart. Then a motorized Agena unit pulled the Gemini inward. Once the two craft were tightly moored, matching electrical contacts met and gave the Gemini astronauts direct control of Agena's onboard equipment - guidance, propulsion, attitude control, relay switches, and the rest. Union of the two vehicles results in a Gemini /Agena spacecraft almost 50 feet long and much more versatile. The rocket power provided by Agena allowed for flights to higher altitudes or to change orbital plane.” [3]

\section{B. Apollo}

Many Gemini crewmembers went on to become Apollo crewmembers. Fabrication of the first Apollo spacecraft began in August 1964. The Apollo program was interrupted by a fire on the launch pad of Apollo 1 on January 27, 1967 [4], which killed the three crewmembers, Ed White, Gus Grissom, and Roger Chaffee. When the Apollo program resumed, the first manned mission was named Apollo 7. Apollo 9 was the first Apollo flight to dock, which occurred on March 3, 1969. The Apollo docking mechanism was also a probe \& drogue mechanism. A view of this mechanism is shown in Figure 3.

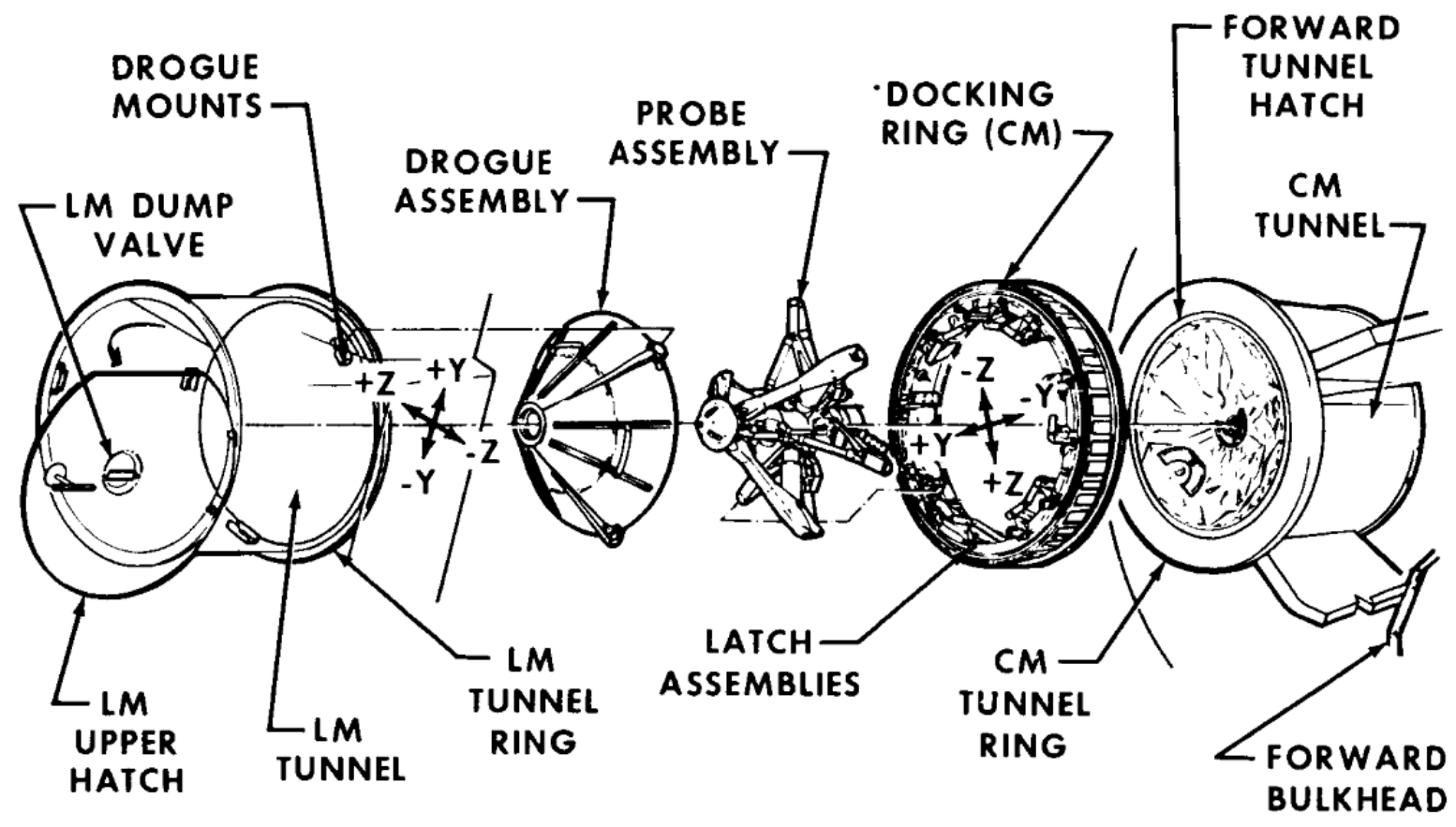

Fig. 3, View of Apollo Docking Mechanism Image courtesy of NASA [5] 
An overview of the Apollo spacecraft and their mechanisms is shown in Figure 4.

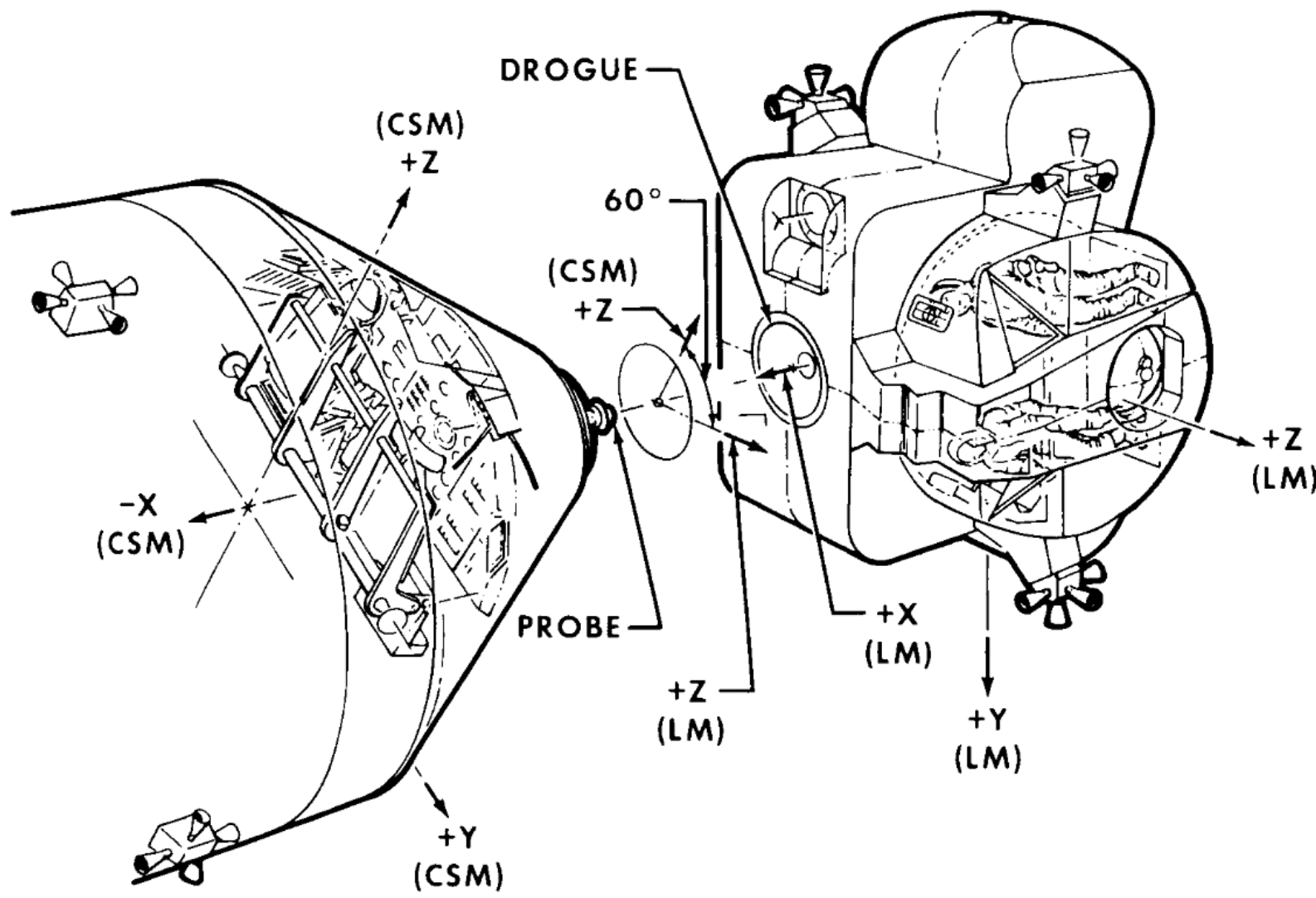

Fig. 4. Overview of Apollo spacecraft

Image courtesy of NASA [6]

During the Lunar Module (LM) extraction from the Saturn Upper Stage S-IVB in Low Earth Orbit, the command module acted as the active spacecraft and the LM acted as the passive spacecraft. During the rendezvous in Lunar Orbit, the LM acted as the active spacecraft and the Command/Service Module (CSM) acted as the passive spacecraft. To begin the docking process, the CSM probe was extended. Docking was accomplished by maneuvering the probe into the cone shaped drogue. The cone shape guided the probe tip to the apex of the cone, where three capture latches achieved soft capture. The crew then retracted the probe, engaging 12 hard capture latches around the perimeter of the docking tunnel which structurally sealed the interface between vehicles. The probe was then removed when needed to allow for crew transfer. 


\section{Soyuz}

The Russians’ first docking was the docking of a Soyuz with another Soyuz on January 4, 1969. The Russian Docking Mechanism was a Probe \& Drogue Mechanism. This mechanism is still in use today. Like Apollo, it is characterized by a central probe, which enters a cone shaped receptacle and is soft captured by latches at the end of the probe. A view of this mechanism is shown in Figures 13 and 14 . Early variants of this mechanism did not allow for transfer of crew between modules. Later variants had hinges which allowed the probe and drogue to be rotated out of the way to allow for crew transfer between modules.

\section{Salyut}

The First Russian Space Station was Salyut. The Russians launched the first module of the Salyut station on April 19 1971. The first Soyuz docking with Salyut was on June 30, 1971. [7] This also used the Probe \& Drogue mechanism.

\section{E. Skylab}

Skylab was the first American space station and this program used Apollo derived hardware. Skylab 1 was launched on May 14, 1973. The living quarters of this space station were derived from an empty third stage (SIVB) of the Saturn V rocket. The docking mechanism between the Apollo Command/Service Module (CSM) and the Apollo Docking Module was an Apollo Probe \& Drogue. An image of the Skylab is shown in Figure 5. 


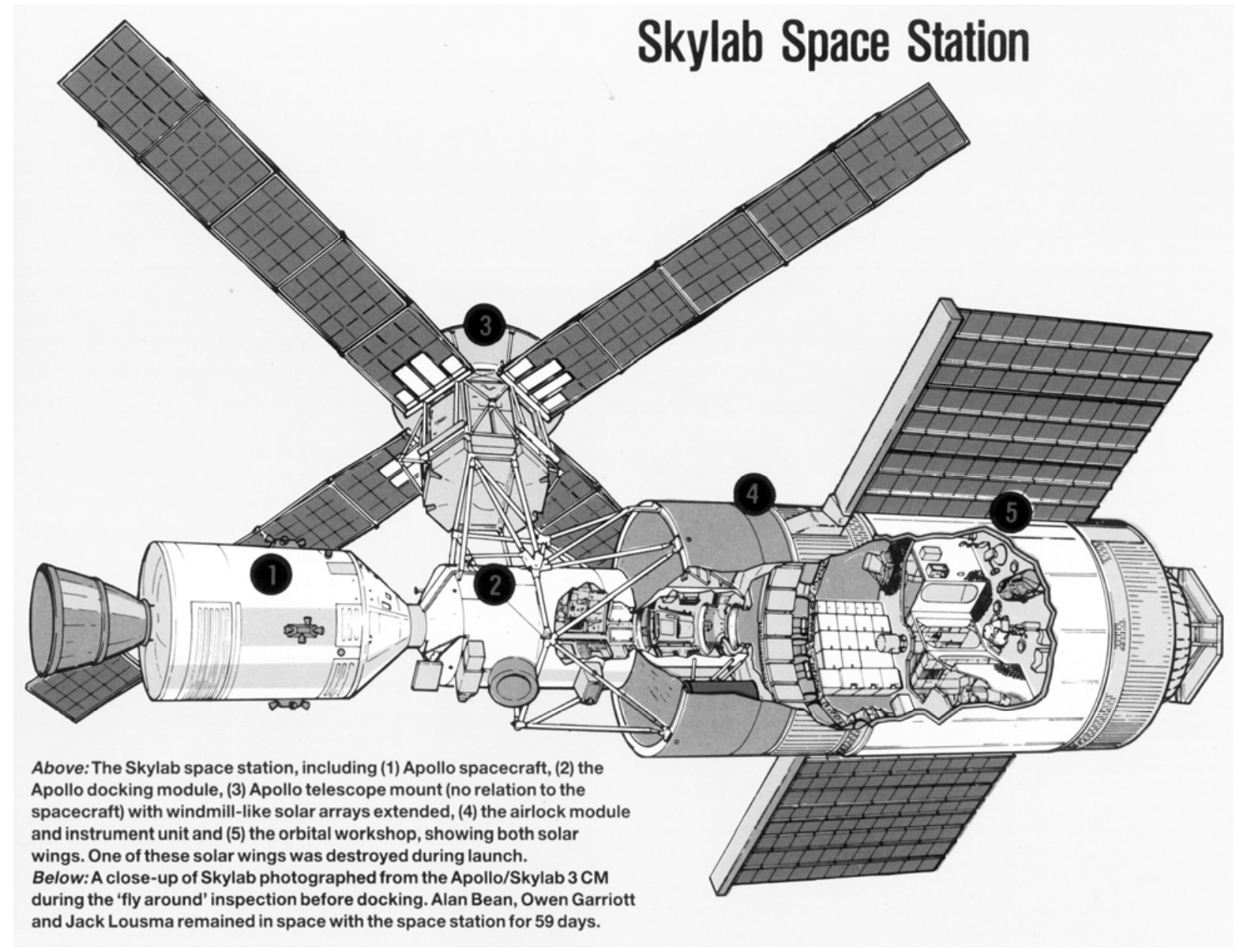

Fig. 5 View showing Skylab Space Station [8]

\section{F. Apollo-Soyuz}

The Apollo/Soyuz project was the first cooperation between two previously adversarial nations; America and the then Soviet Russia. This program marked a turning point in the relations between the two nations and laid the groundwork for Shuttle/Mir and the International Space Station. Apollo Soyuz project launched on July 15, 1975 and docked on July 17, 1975. The spacecraft used in the project were the Apollo Command/Service Module (CSM) spacecraft and the Soyuz. A docking module was created and launched, attached to the Command Module, which provided the interface between the two vehicles. An overview of the two vehicles is shown in Figure 6. A detail view of the USSR and US side of the Apollo/Soyuz mechanism is shown in Figure 7 and Figure 8. 


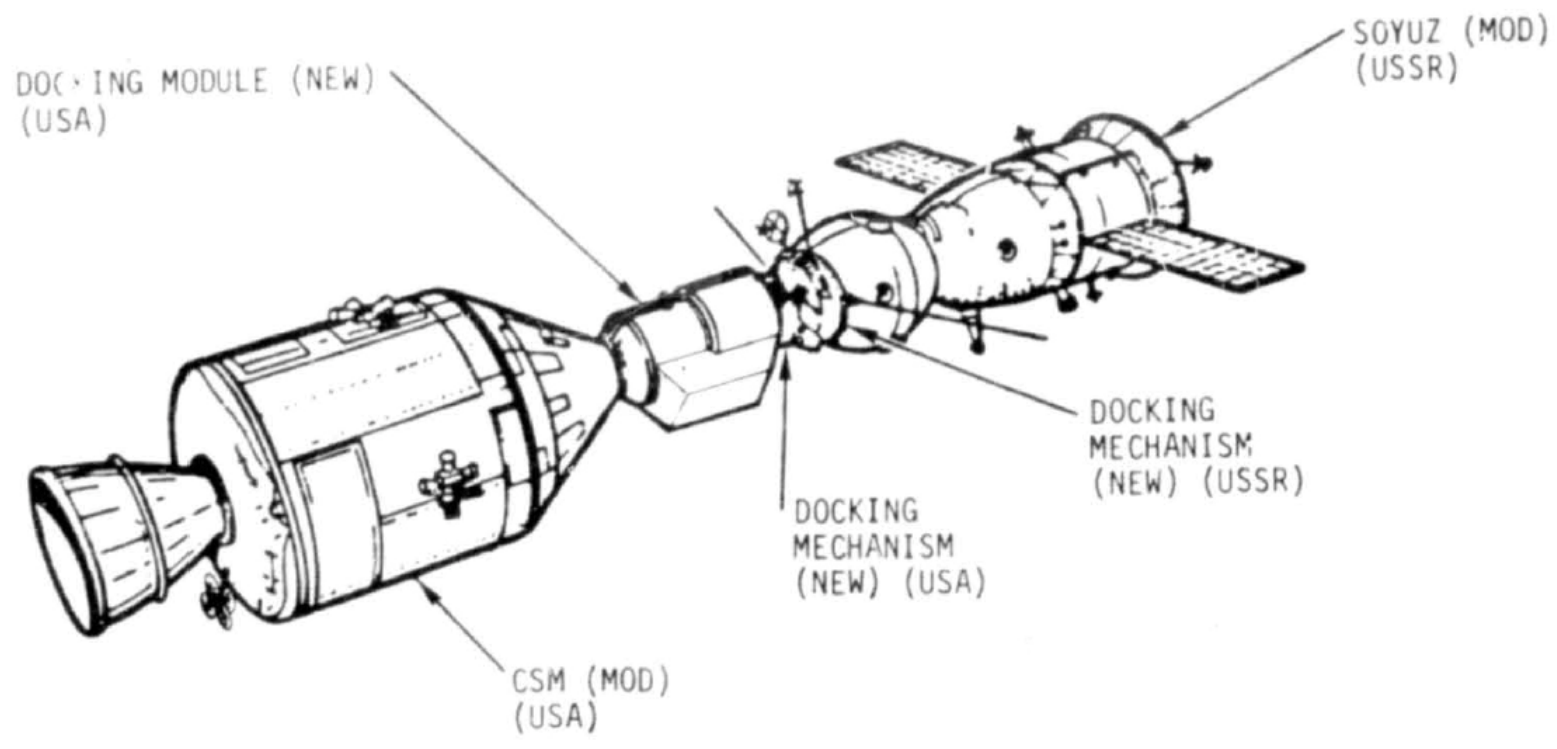

Fig. 6 View of Apollo/Soyuz Vehicles

Image courtesy of NASA [9]

The Apollo/Soyuz mechanism was an androgynous mechanism. This concept was that, in the docking process, one vehicle's mechanism would be acting as an active mechanism, with the guide ring extended, while the other vehicle's mechanism would be acting in the passive manner with the ring retracted. Either mechanism could perform the active or passive docking function. 


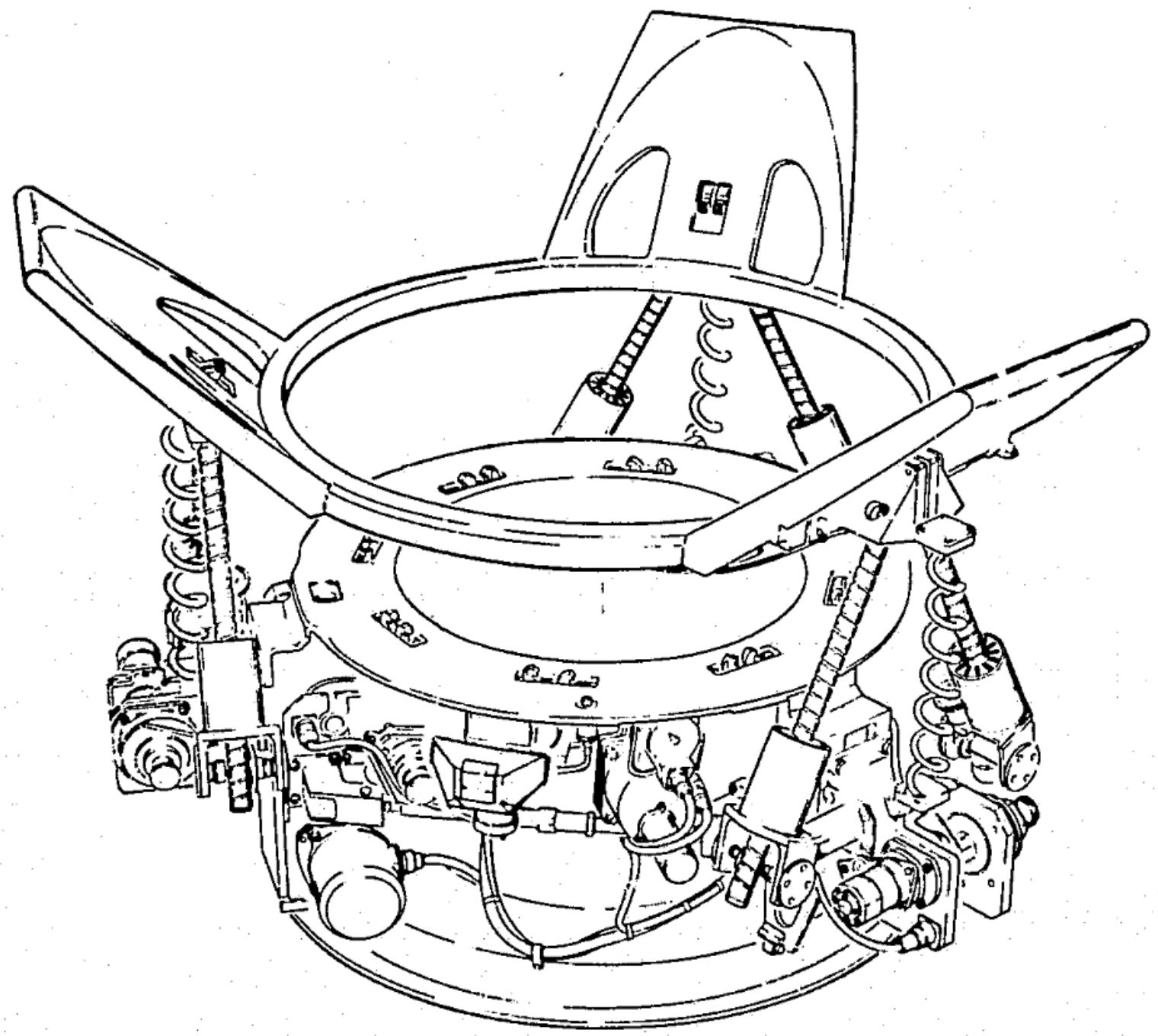

Fig. 7 Detail view of the USSR half of the Apollo Soyuz Mechanism Image courtesy of NASA [10] 


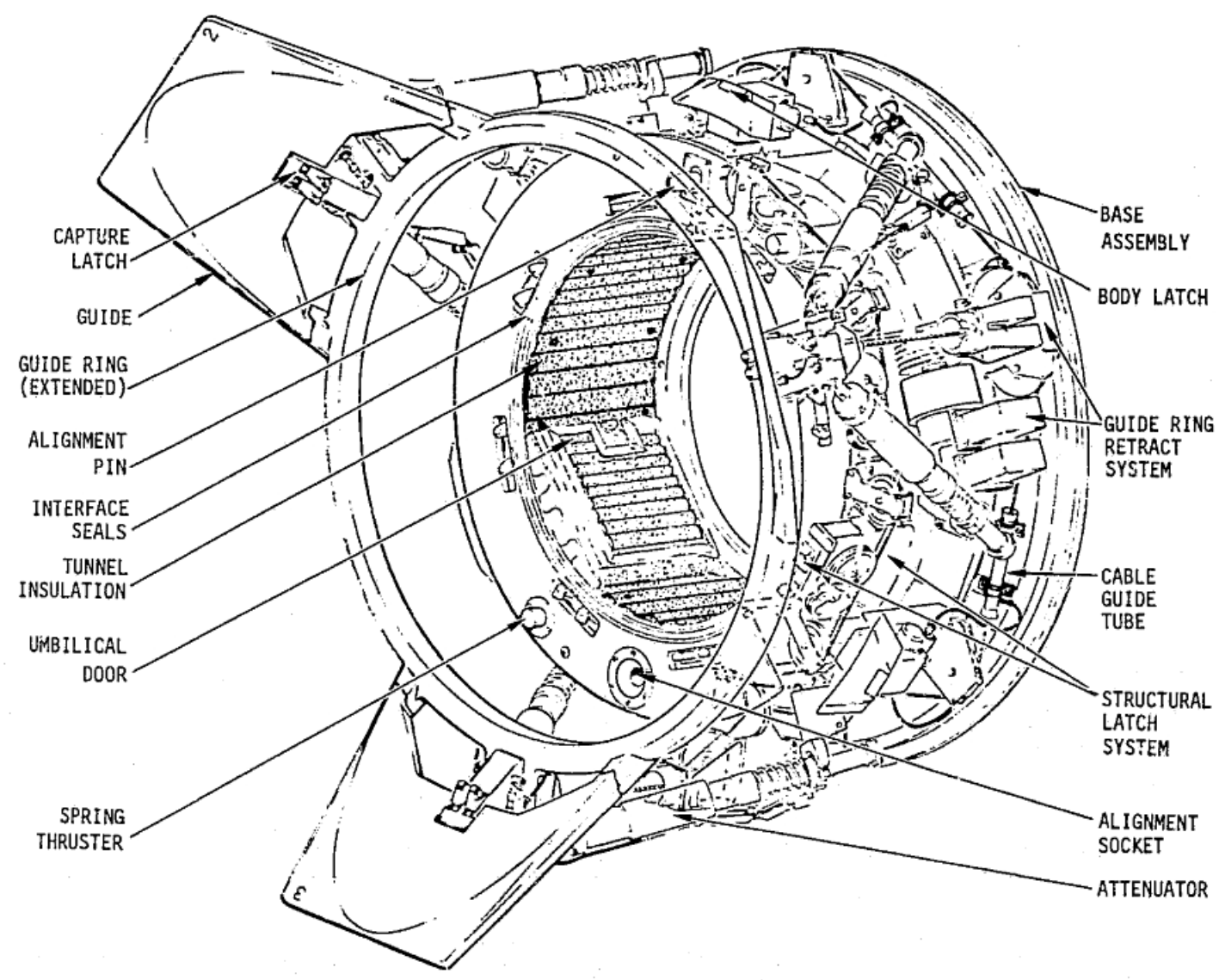

Fig. 8 Detail View of the U.S. potion of the Apollo Soyuz docking mechanism Image courtesy of NASA [11]

\section{G. Mir}

The on-orbit assembly of the Russian space station Mir began on February 19, 1986. Mir was launched in a plane inclined $51.6^{\circ}$ to the equator, as is the International Space Station. Mir had two docking ports and four berthing ports. Mir primarily used the Russian Probe \& Drogue docking mechanism with the addition of one Androgynous Peripheral Attachment System (APAS), to be used with Mir/Shuttle. A view of Mir is shown in Figure 9. The APAS docking module, to which the shuttle would dock, is the orange colored module on the right of the Figure. 


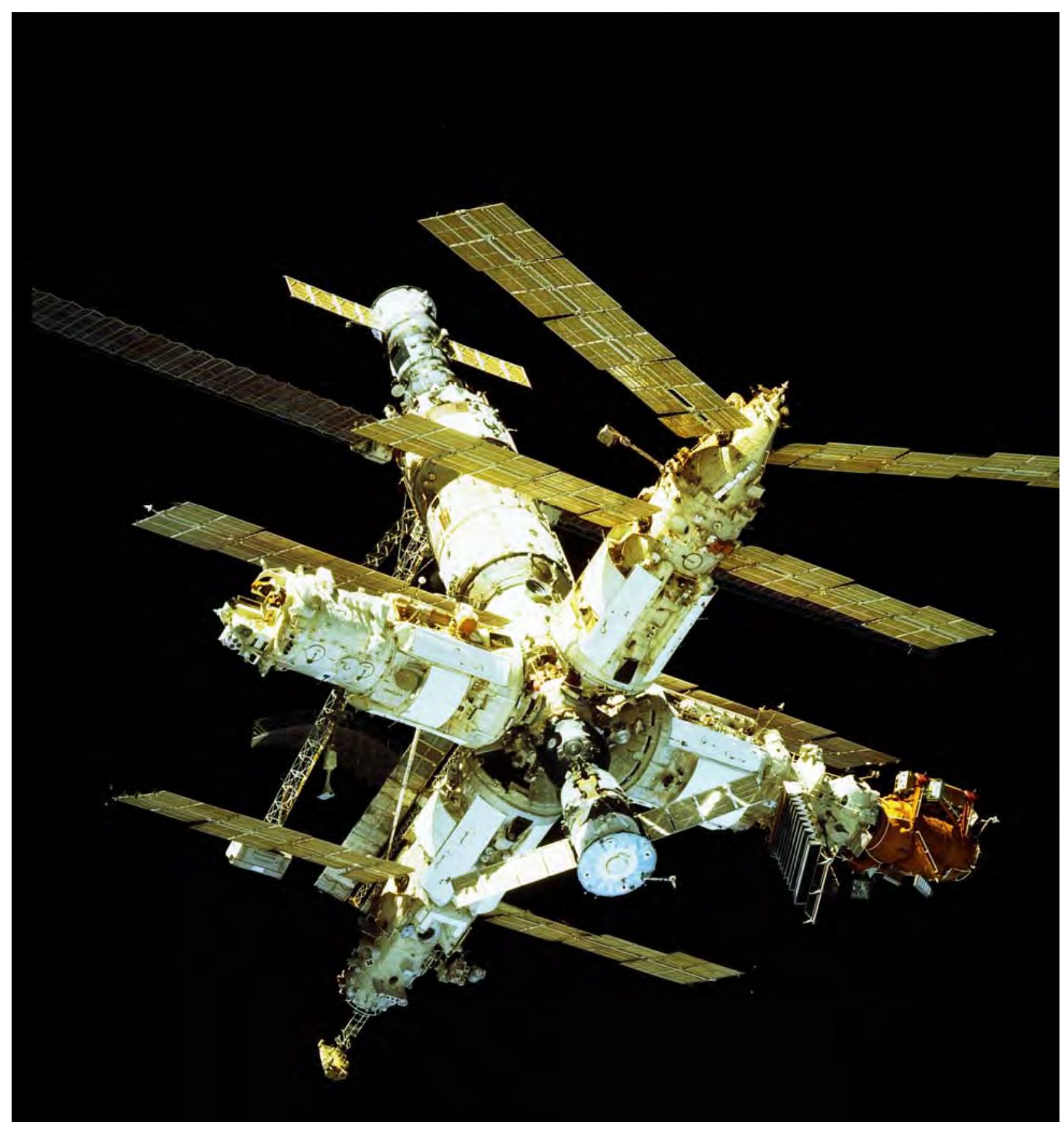

Fig. 9 View showing Mir Space Station

Image courtesy of NASA [12]

\section{H. Mir/Shuttle}

The Mir/Shuttle program was the prelude to the international cooperation that made possible the International Space Station. Working together, docking the Shuttle to Mir and flying crews to Mir allowed for more experience operating cooperatively and allowed the Russian and American teams to adapt to each other's distinct operational 
methods and cultures. The docking interface between the Shuttle and Mir was an APAS. This was a later evolution of the androgynous docking mechanism used on Apollo-Soyuz. Whereas Apollo-Soyuz had outward facing petals, the APAS89 had inward facing petals. The mounting of the soft capture mechanisms on the periphery of the docking interface meant crews could immediately transfer between vehicles without having to remove a bulky central Probe \& Drogue mechanism first. The shuttle APAS is shown in Figure 10.

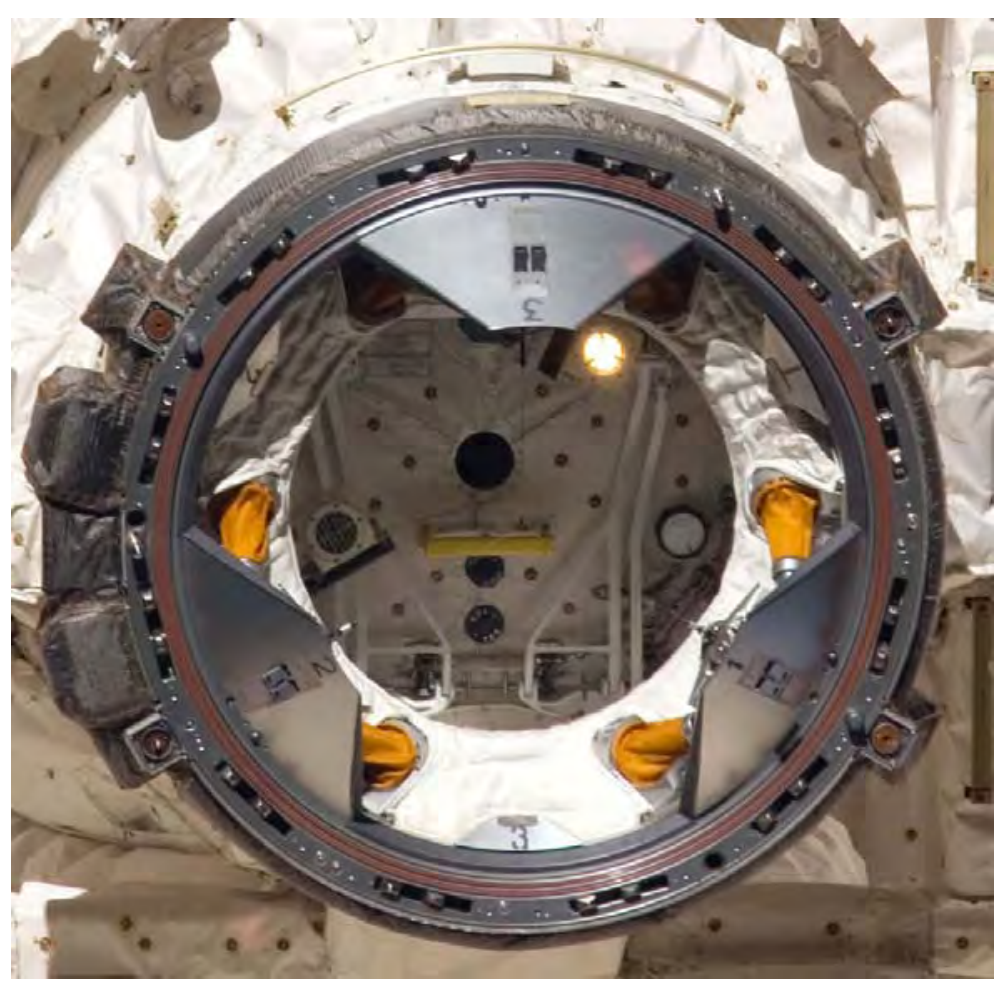

Fig. 10 View Showing APAS on the Orbiter Docking System Image courtesy of NASA [13] 


\section{International Space Station}

The first core module of the International Space Station (ISS) was launched on November 20, 1998. It has since grown to become the largest space station ever built. It has a number of berthing and docking mechanisms. These will be discussed more in following chapters. A view of the ISS is shown in Figure 11.

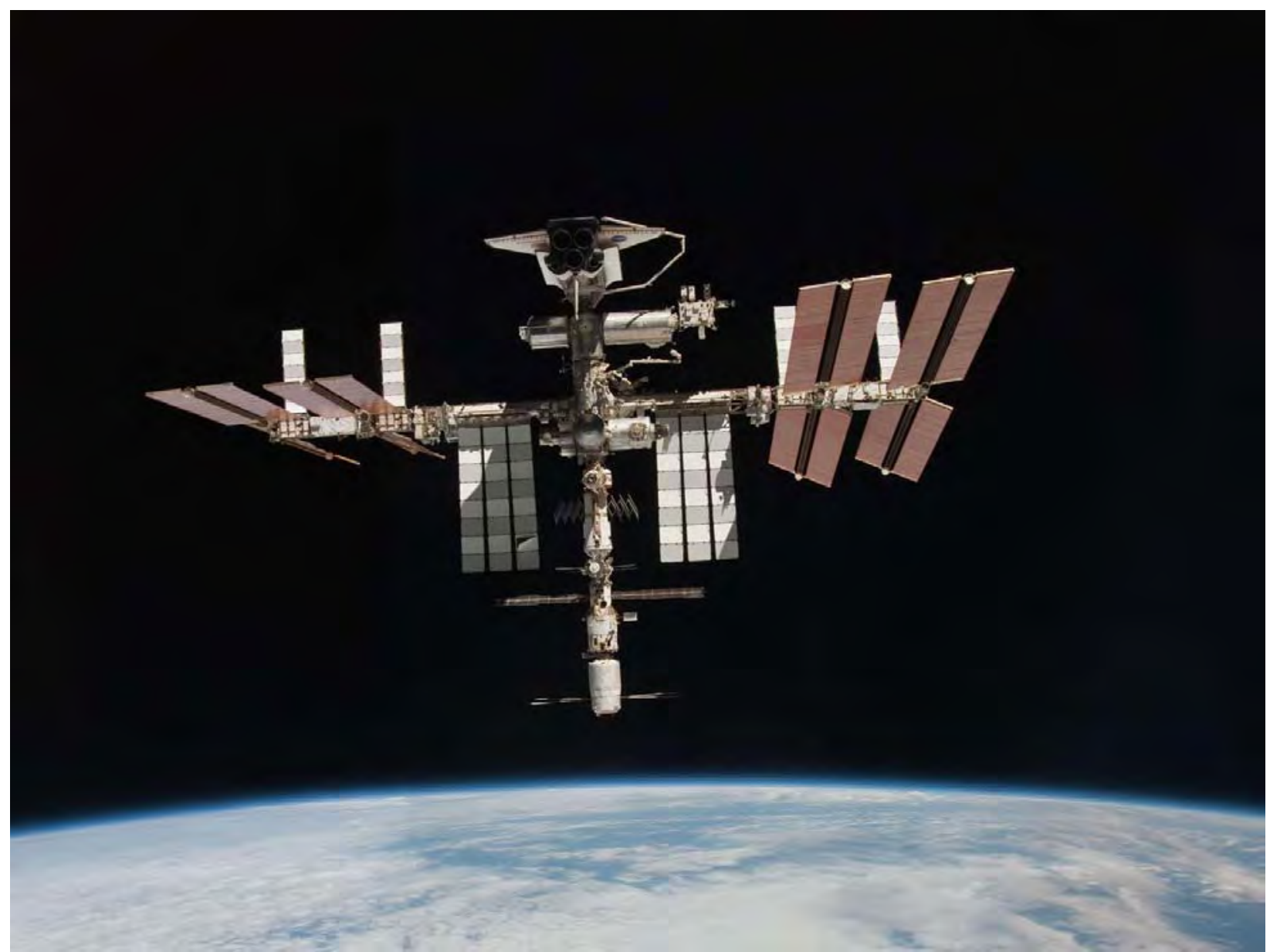

Fig. 11 View showing the International Space Station Image courtesy of NASA [14] 
The International Space Station has been a testbed for berthing and docking mechanisms. The ISS is being assembled from components made around the world by multiple contractors. There are many different interfaces between countries and contractors. These interfaces have taken many different forms, some pressurized to join modules where the crew will live and work, and some unpressurized to join the primary structural elements together, along with robotic workstations and logistics carrier elements. These forms have served the different purposes for interfaces between elements. The first mating interface used was the APAS95 between the U.S. Pressurized Mating Adapter 1 and the Russian FGB. The next interface was between the FGB and the Service Module. This mechanism was the Hybrid docking mechanism. The next interface type was between Z1 truss and Node 1. This interface used a Common Berthing Mechanism, created by Boeing. This mechanism type was used repeatedly for the U.S. segment, but the rest of the unique interface types were unpressurized. The next interface type was between the P6 truss and the Zenith bulkhead of Z1. This interface was a Rocketdyne Truss Attachment System (RTAS). An additional interface is between the Truss segment S0 and a pressurized segment, the U.S. Lab. This mechanism is the Module to Truss Segment Attachment System (MTSAS). The next interfaces were between Truss Segments, such as the Segment to Segment Attachment Mechanism (SSAS). The next type of interface was Common Attachment System (CAS). This was first used between ESP3 logistics carrier and the P3 zenith CAS site. The next type of interface was a Japanese interface, the Exposed Facility Berthing Mechanism (EFBM), between the Exposed Facility (EF) and the Japanese Pressurized Module (PM). An additional Japanese interface mechanism is the Exposed Facility Unit (EFU), between the Exposed Segment (ES) and the EF. All of these mechanisms are in the ISS generation of mechanisms between 1998 and 2009. An overview of all of the mechanisms is shown in Figure 12. 


\section{ISS Element to Element Interface Topology}

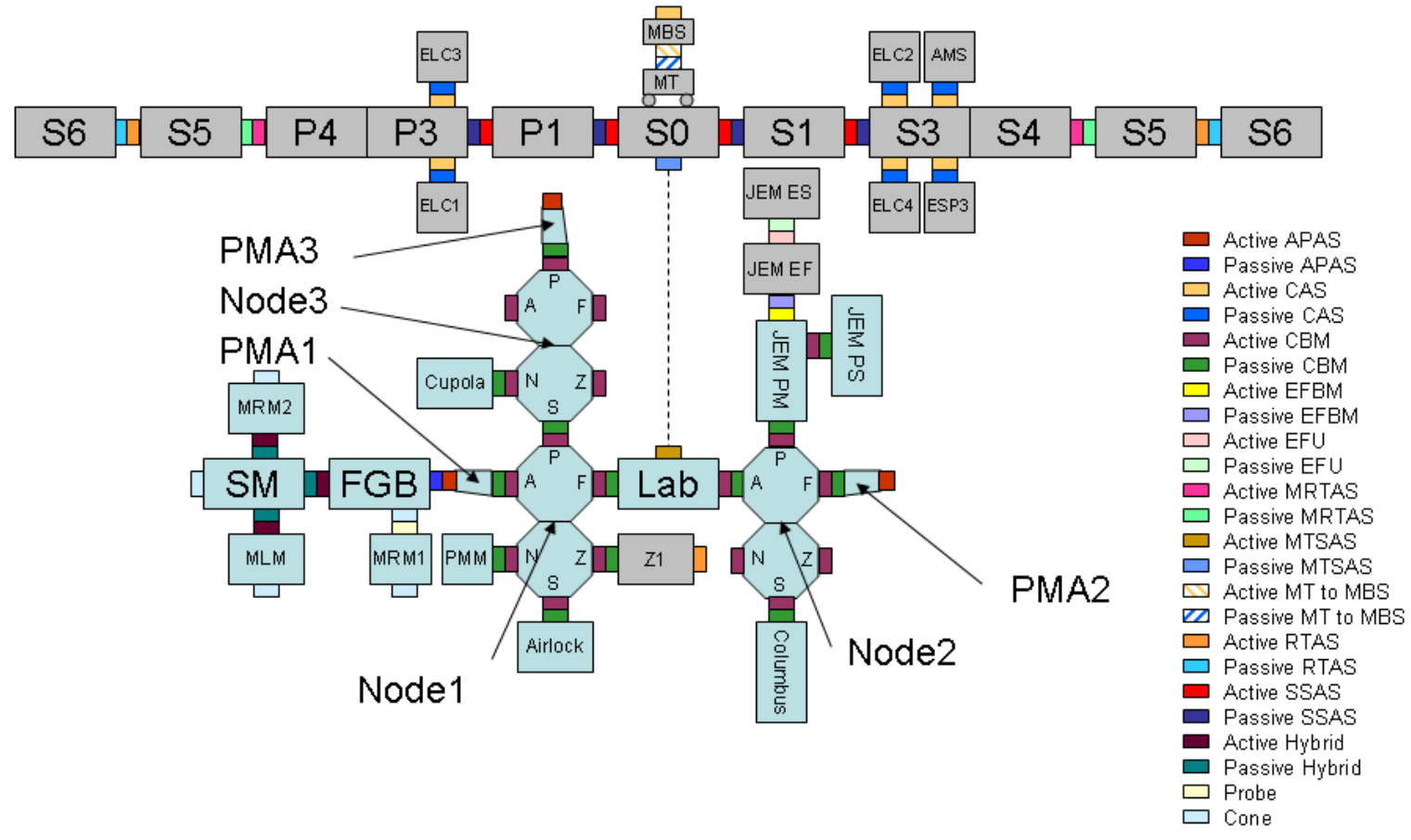

Fig. 12 View Showing ISS Element to Element Interfaces 


\section{Anatomy of Pressurized Mechanisms}

\section{J. Probe \& Drogue}

The Russian Probe \& Drogue is made up of two halves; a Probe and a Drogue, or cone. The Probe enters the Drogue and the soft capture latches engage a receptacle at the apex of the cone shaped Drogue. The Probe is then retracted, allowing coarse alignment to occur followed by the seating of the fine alignment features and closing of the eight hard capture latches. A view of the Probe \& Drogue mechanism is shown in Figures 13 and 14.

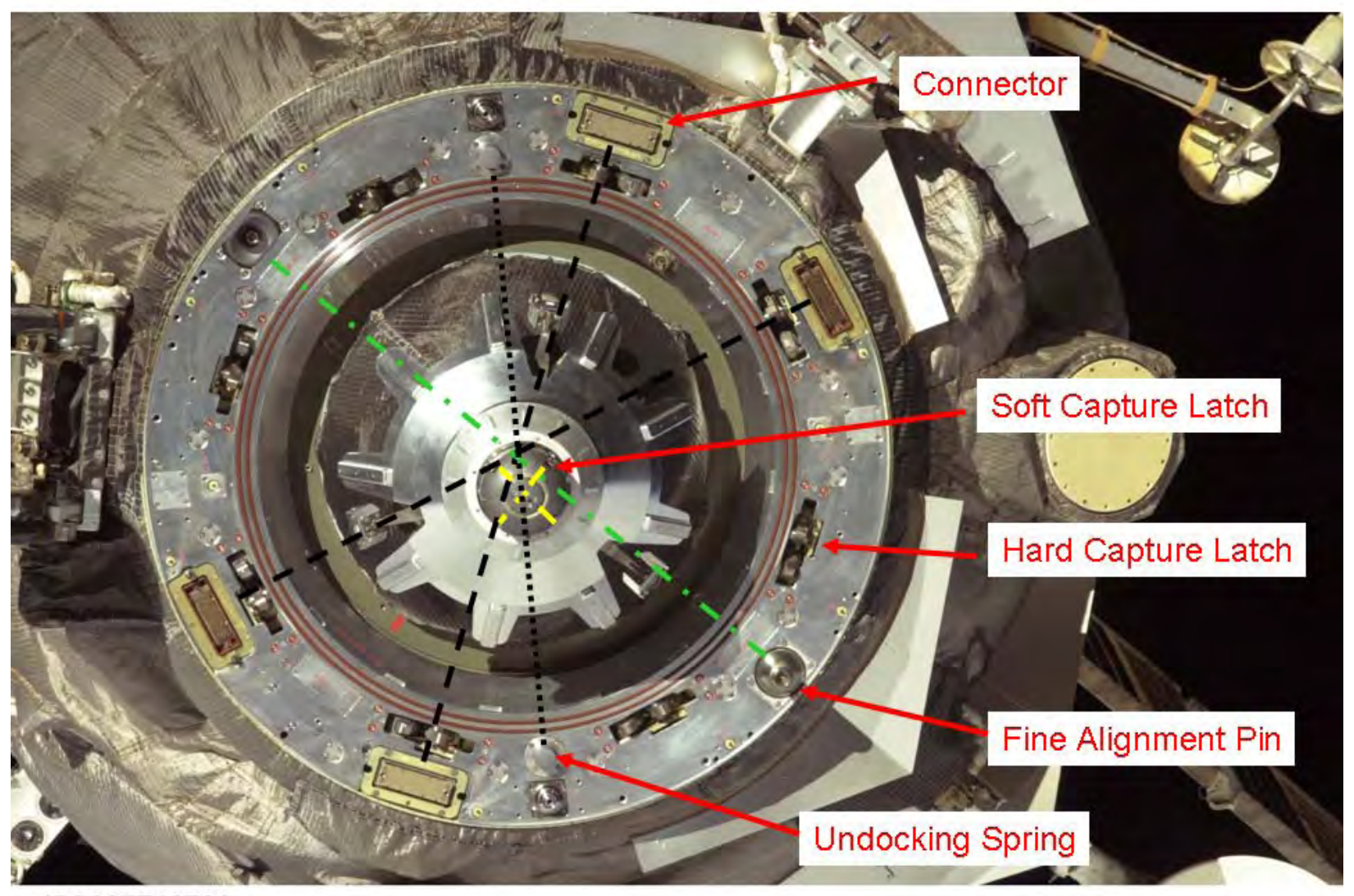

ISS007E13791

Fig. 13 View showing Probe half of Probe \& Drogue interface Image courtesy of NASA [15] 


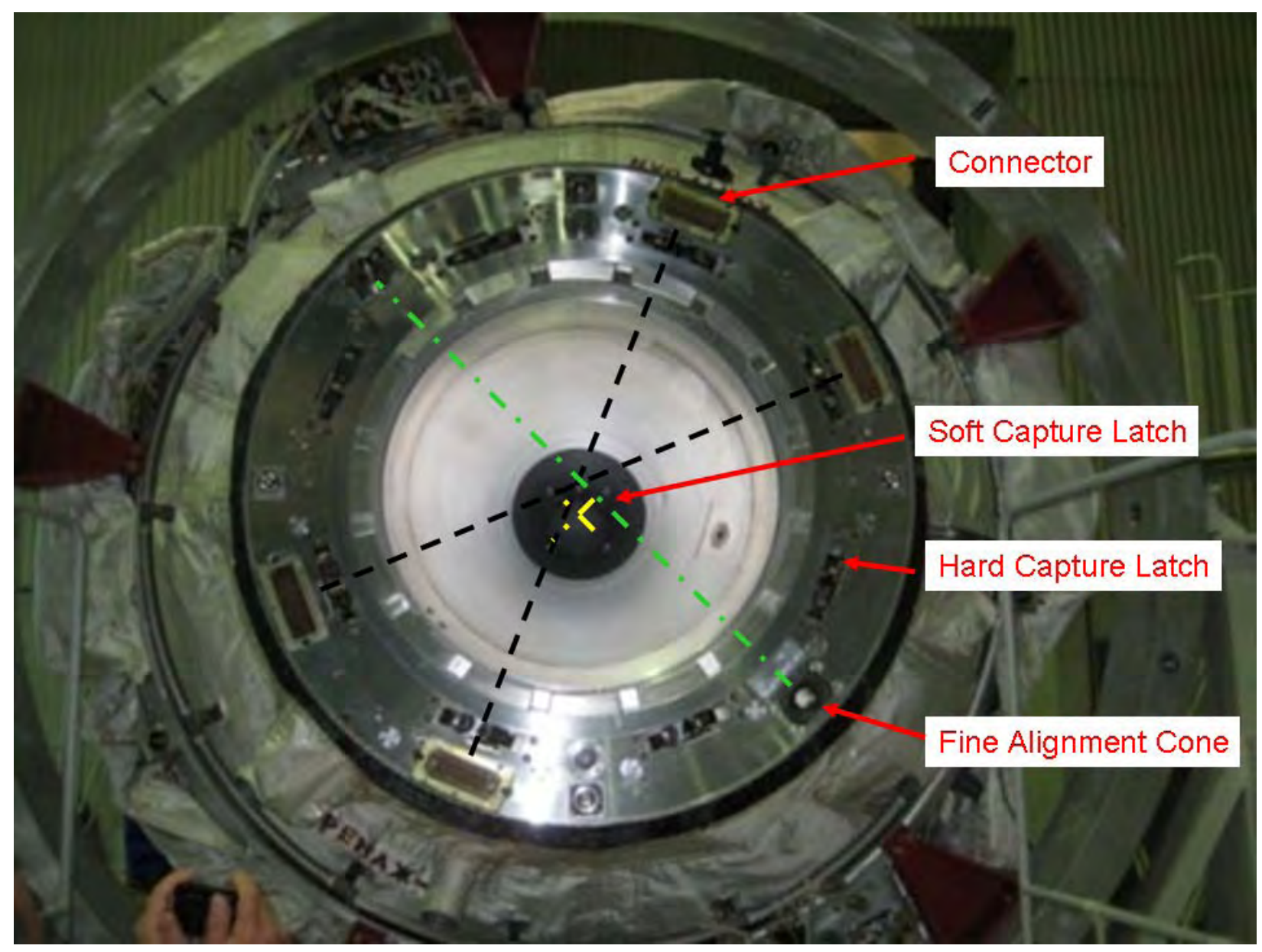

Fig. 14 View Showing Drogue half of Probe \& Drogue interface [16] 


\section{K. Hybrid}

The Russian Hybrid interface mechanism is a cross between an APAS and a Probe \& Drogue interface. There is a Probe \& Drogue soft capture interface which acts the same as the Probe \& Drogue interface, but the hard capture is the same as the APAS with twelve capture latches and the same fine alignment features, undocking springs and interface sensors. Views of the hybrid mechanism are shown in Figures 15 and 16.

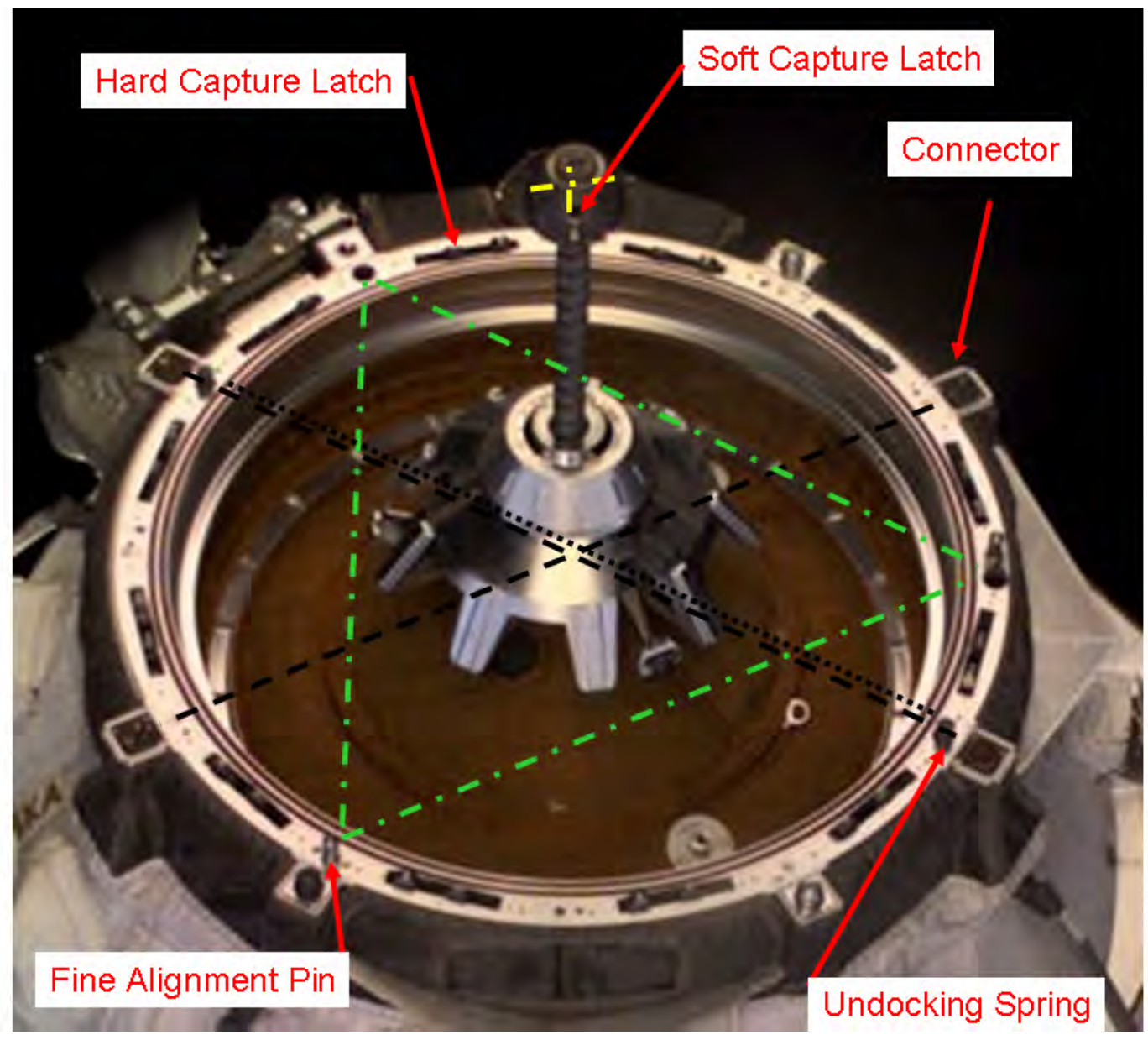

Fig. 15 View Showing Probe half of Hybrid Interface [17] 


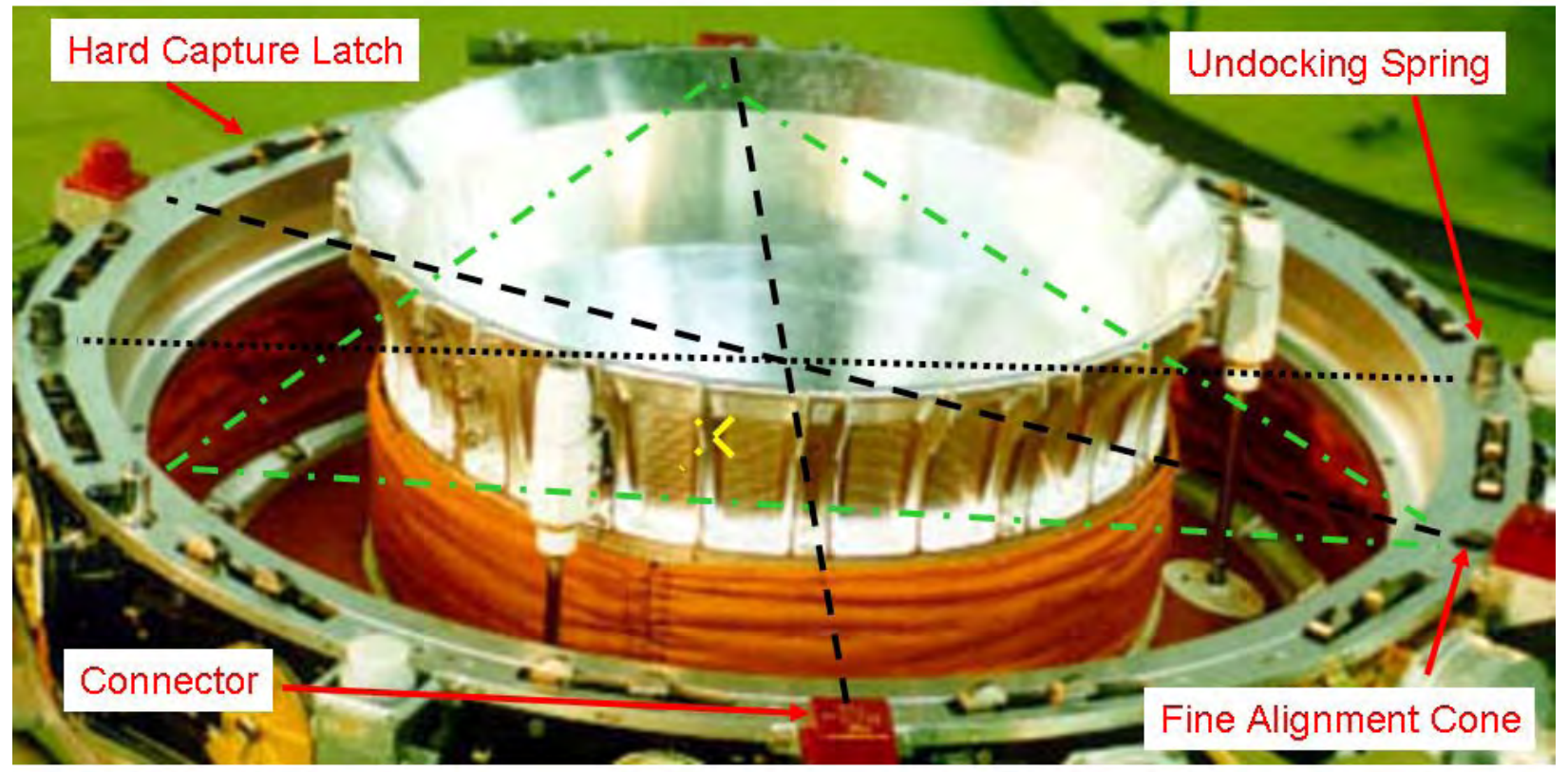

Fig. 16 View showing Drogue half of Hybrid interface [18]

\section{Androgynous Peripheral Attachment System (APAS)}

The APAS is, by its name designed to be androgynous, meaning both halves of the mechanisms used on both mating vehicles were identical and either side could act in the active or passive docking role. However on the ISS Pressurized Mating Adapters (PMAs) the station half of the APAS is permanently passive, meaning that it has a fixed guide ring and the shuttle APAS was assumed to act in the active role always, with its guide ring initially extended for soft docking. Figure 17 shows an APAS with the guide ring extended. This extended guide ring allows for a soft capture of the docking interface where relative lateral and angular misalignments are taken out, and relative motion is then attenuated, prior to retracting the ring, actuating the structural latches and achieving hard dock. $\quad$ An active and Passive APAS are shown in Figures 17 and 18. 


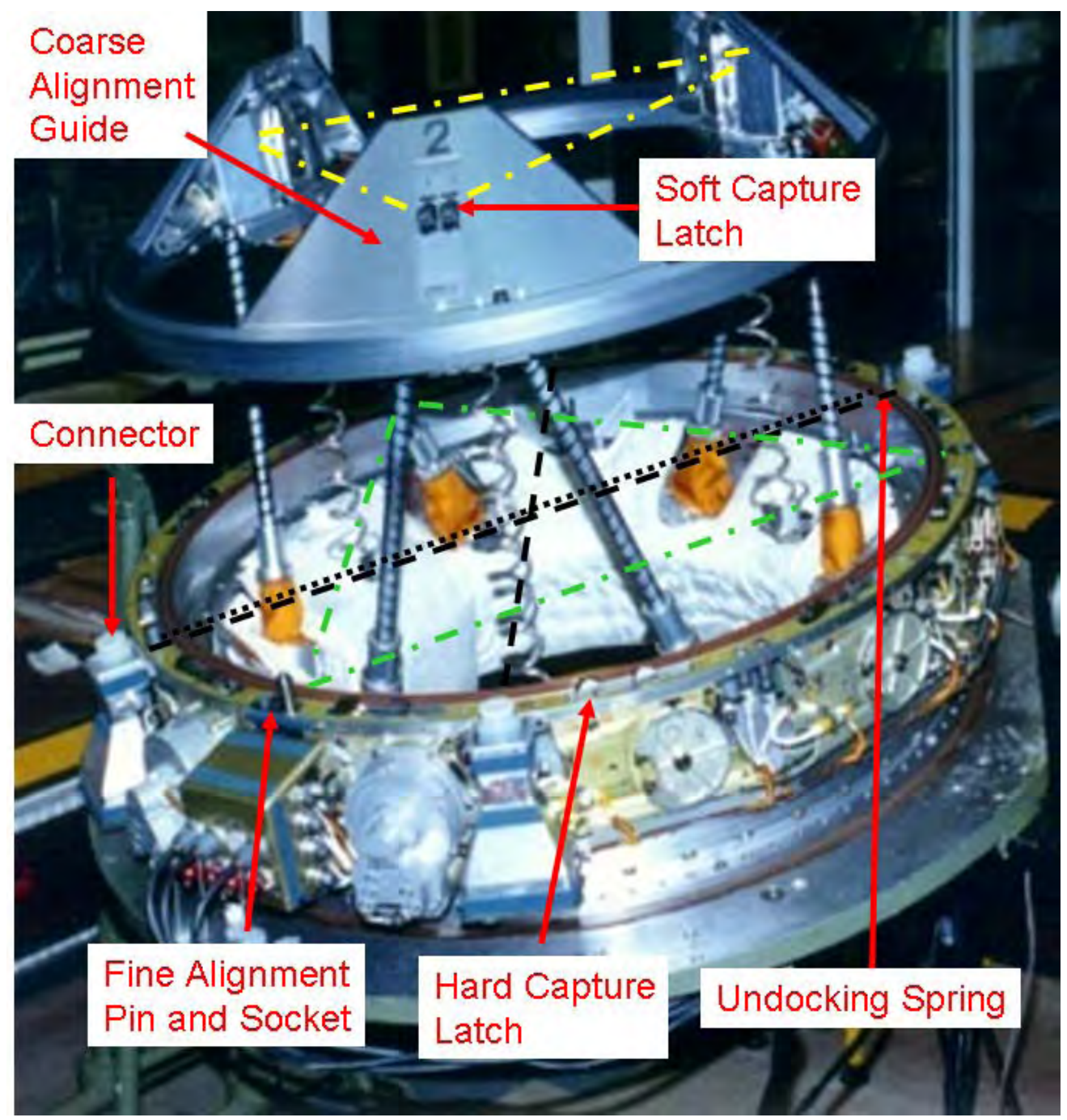

Fig. 17 View showing Active APAS [19] 


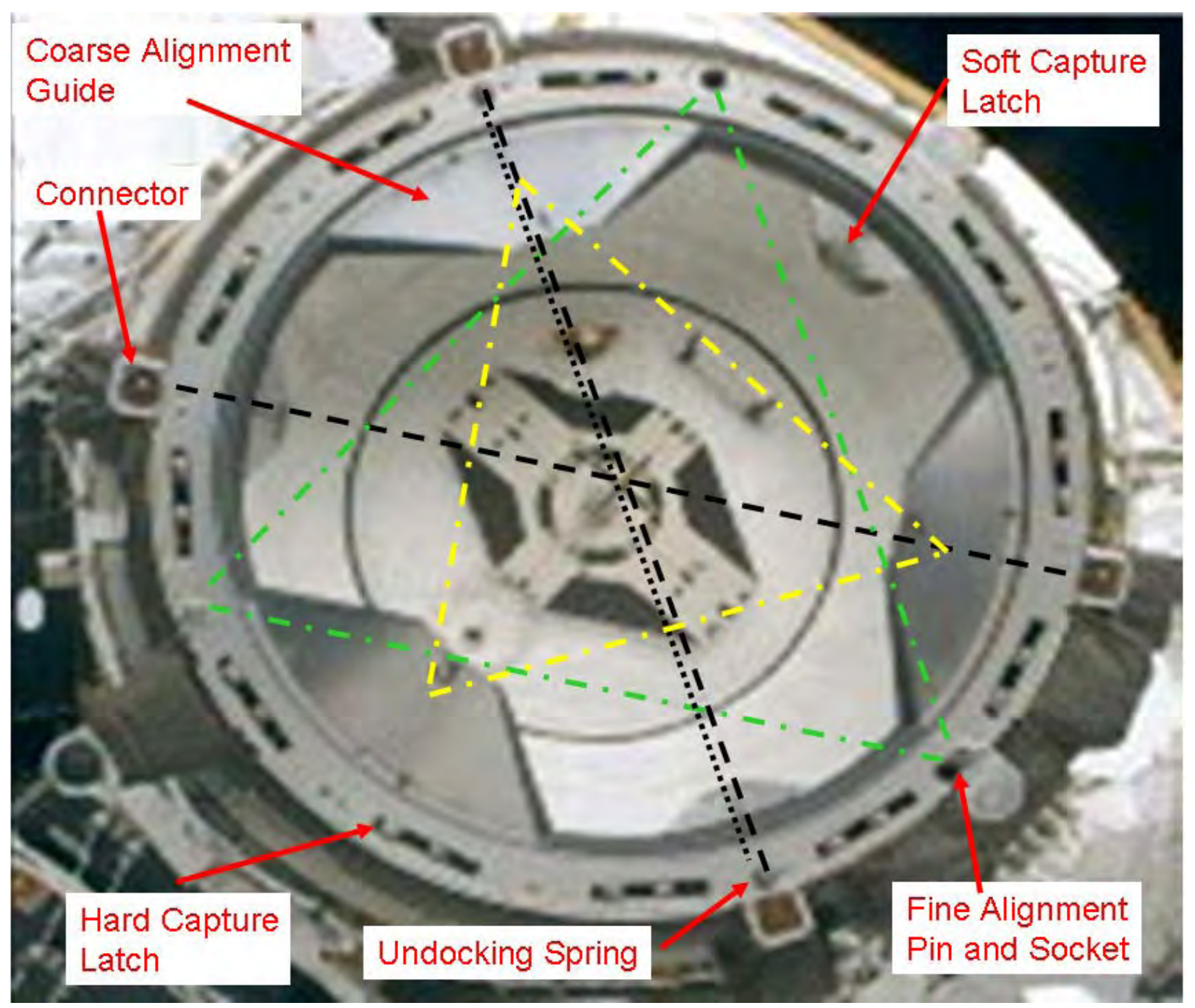

Fig. 18 View3 Showing Passive APAS [20] 


\section{Chinese APAS}

The Chinese APAS is a clone of the Russian APAS. A view of the Chinese APAS is shown in Figure 19.

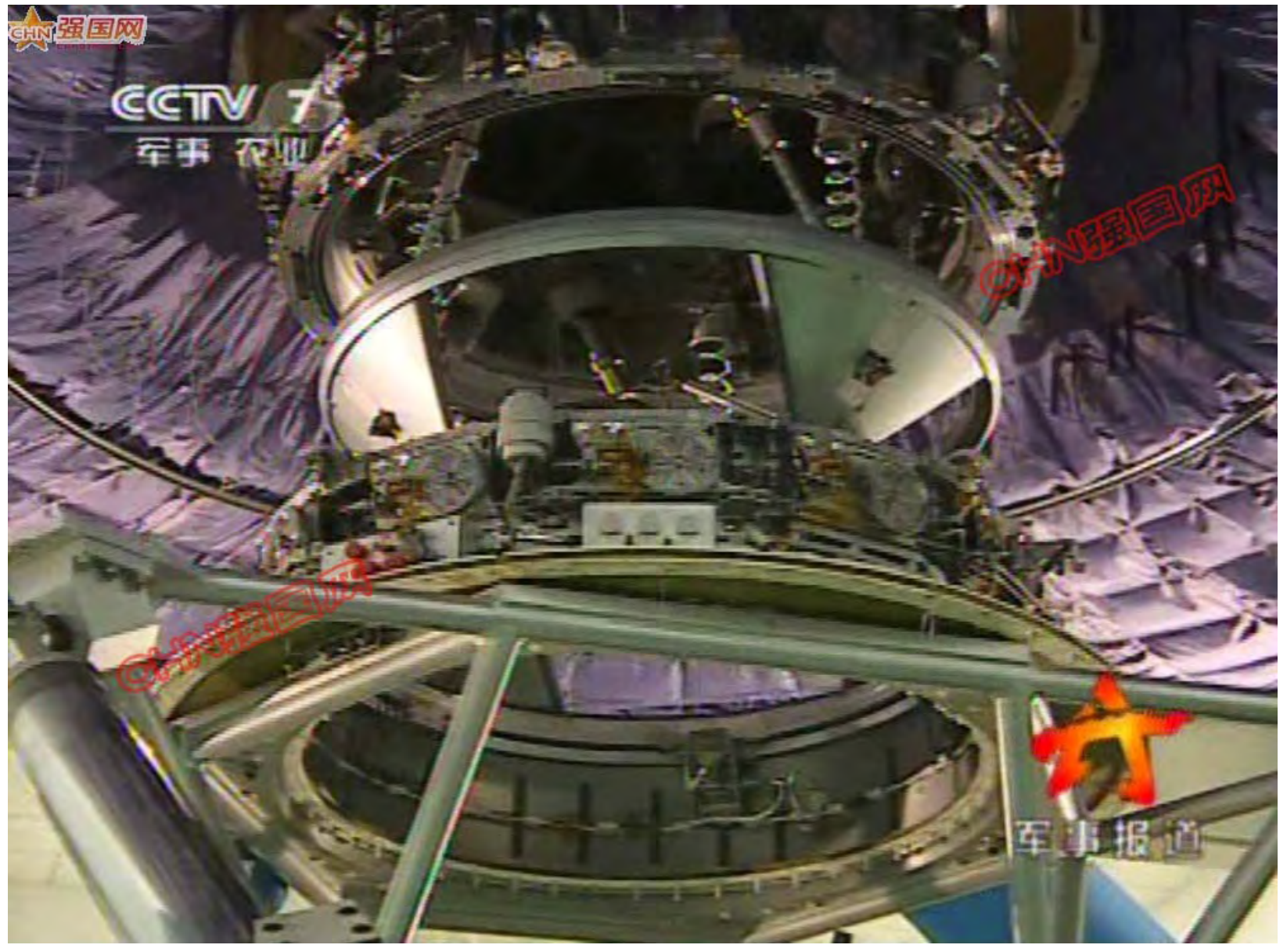

Fig. 19 View Showing Active and Passive Halves of the Chinese APAS [21]

\section{N. Common Berthing Mechanism}

The Common Berthing Mechanism is a Boeing designed product. It is used on all pressurized interfaces of the US segment, with the exception of APASs on the PMAs. The Common Berthing Mechanism (CBM) is used to 
berth between, or with, U.S. pressurized modules. The CBM is made up of two mating halves; an Active (ACBM) and a Passive (PCBM) half. The generic features of the ACBM are:

Coarse Alignment Guides

Ready to Latch Indicators

Capture Latches

Strike Plates

Fine Alignment Pins

Powered Bolts

The generic features of the PCBM are:

Coarse Alignment Guides

Capture Fitting

Thermal Equalization Standoffs

Fine Alignment Sockets

Power Bolt Nuts

When the PCBM is brought into proper alignment with the ACBM (through robotic manipulation of the incoming pressurized element) the coarse alignment guides provide coarse alignment by constraining roll and translation. As the incoming PCBM is translated towards the ACBM, the coarse alignment guides interact with each other as needed and with the ready to latch indicators. When the ready to latch indicators are triggered by the PCBM alignment guides, the capture latches are deployed and draw the PCBM into fine alignment, which is achieved when the ACBM fine alignment pins are seated in the PCBM fine alignment sockets. Once fine alignment is achieved, structural attachment is completed by advancing, first all sixteen and then in groups of four, the 16 powered bolts on the ACBM into the 16 nuts on the PCBM. Views of the ACBM and the PCBM are shown in in Figures 20 and 21. 


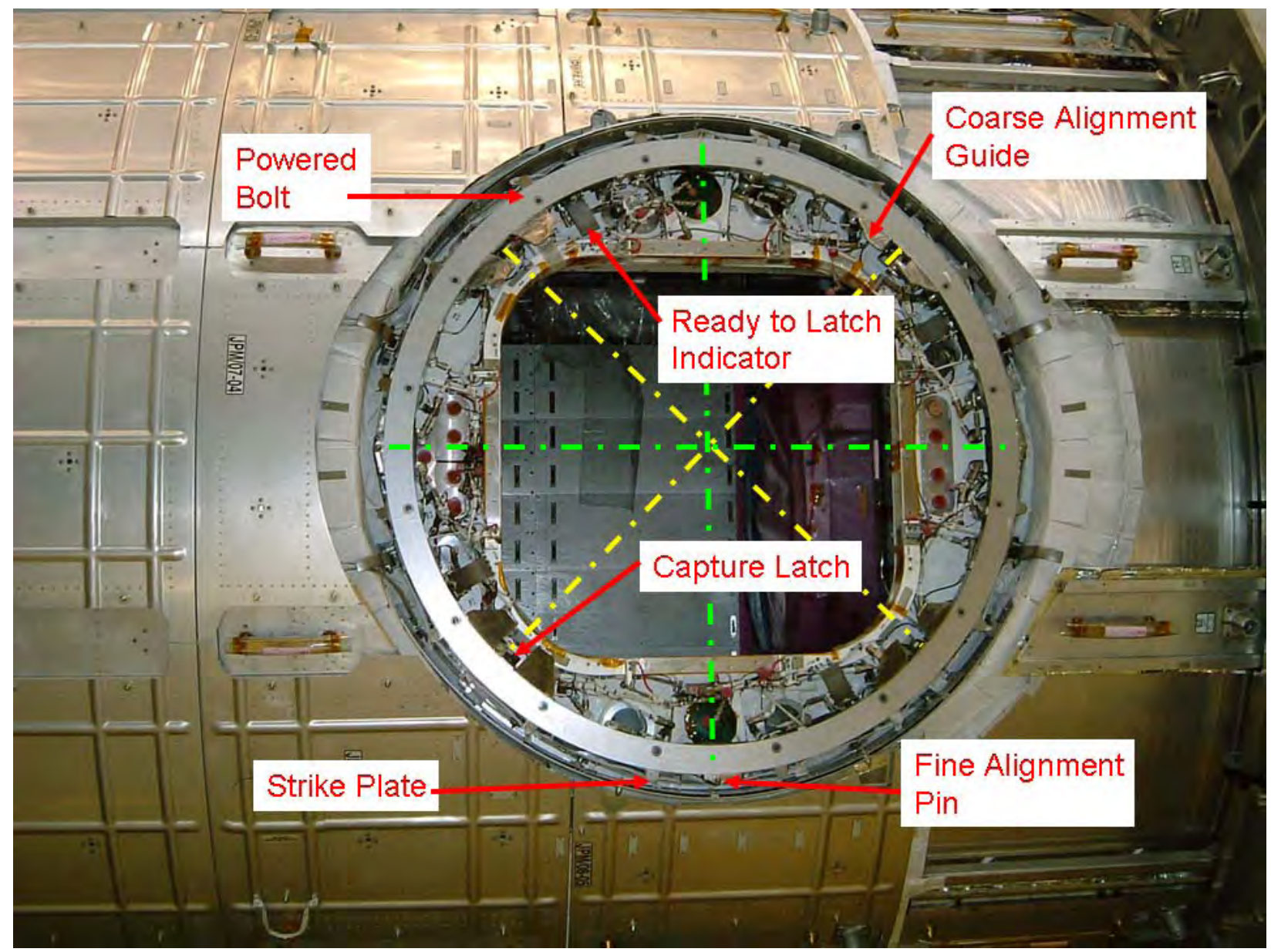

Fig. 20 View showing ACBM

Image courtesy of NASA [22] 


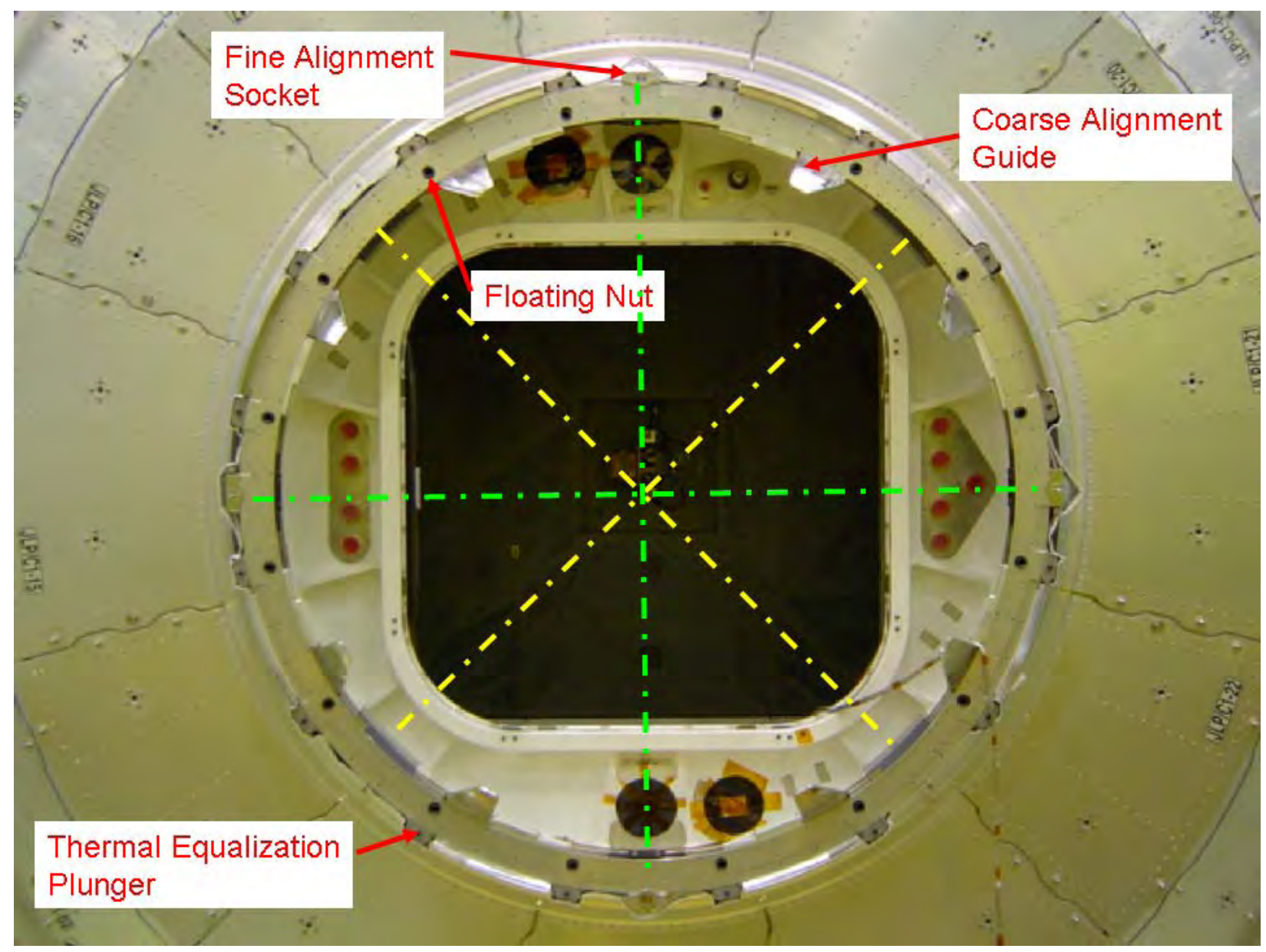

Fig. 21 View showing partial PCBM (seal omitted)

Image courtesy of NASA [23]

\section{Anatomy of Unpressurized Mechanisms}

\section{O. Cup and Cone Alignment with Peripheral Bolts}

This section of mechanisms uses a cup and cone with peripheral bolts. One mechanism of this type is the Segment to Segment Attachment System (SSAS) used on S0, S1. S3, P1 and P3.

1. Segment to Segment Attachment System (SSAS)

An overview of the active SSAS is shown in Figure 17. The SSAS is used to attach inboard Truss segments. The robotic arm operator places the incoming element so that the Ready to Latch (RTL) plungers contact the RTL paddles. When the proper RTL position has been achieved, the claw is closed, capturing the capture bar. 
Meanwhile the three coarse alignment cones adjacent to the three RTL switches and three paddles seat in the three coarse alignment cups. As the claw is further closed, the fine alignment features seat.

The generic features of the active SSAS are the:

Coarse Alignment Guides

Fine Alignment Guides

Ready to Latch Indicators

Capture Latch

The generic features of the passive SSAS are the:

Coarse Alignment Guides

Fine Alignment Guides

Capture Bar

A view of the active SSAS and Passive SSAS are shown in Figures 22 and 23. 


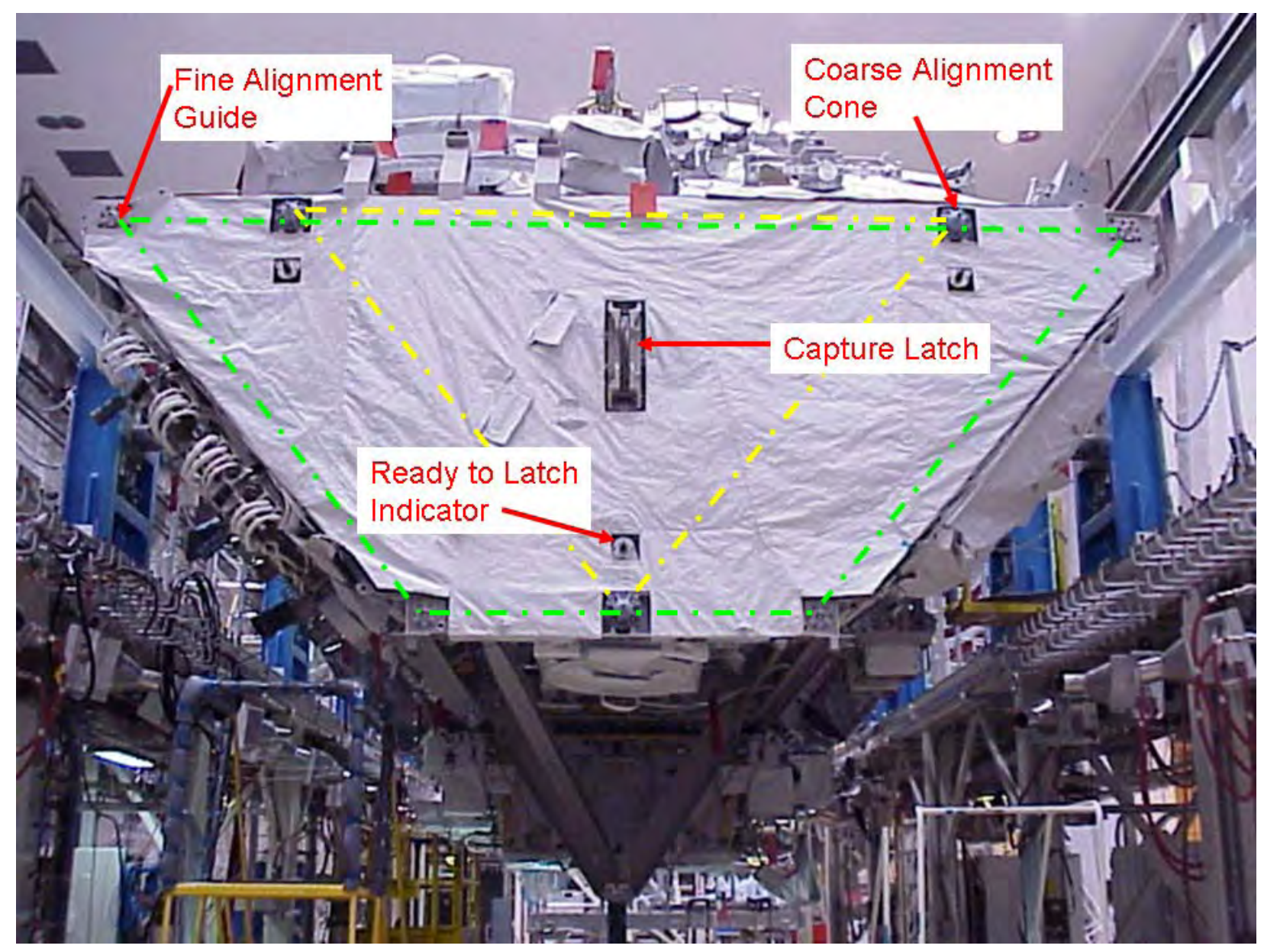

Fig. 22 View Showing Active SSAS [24] 


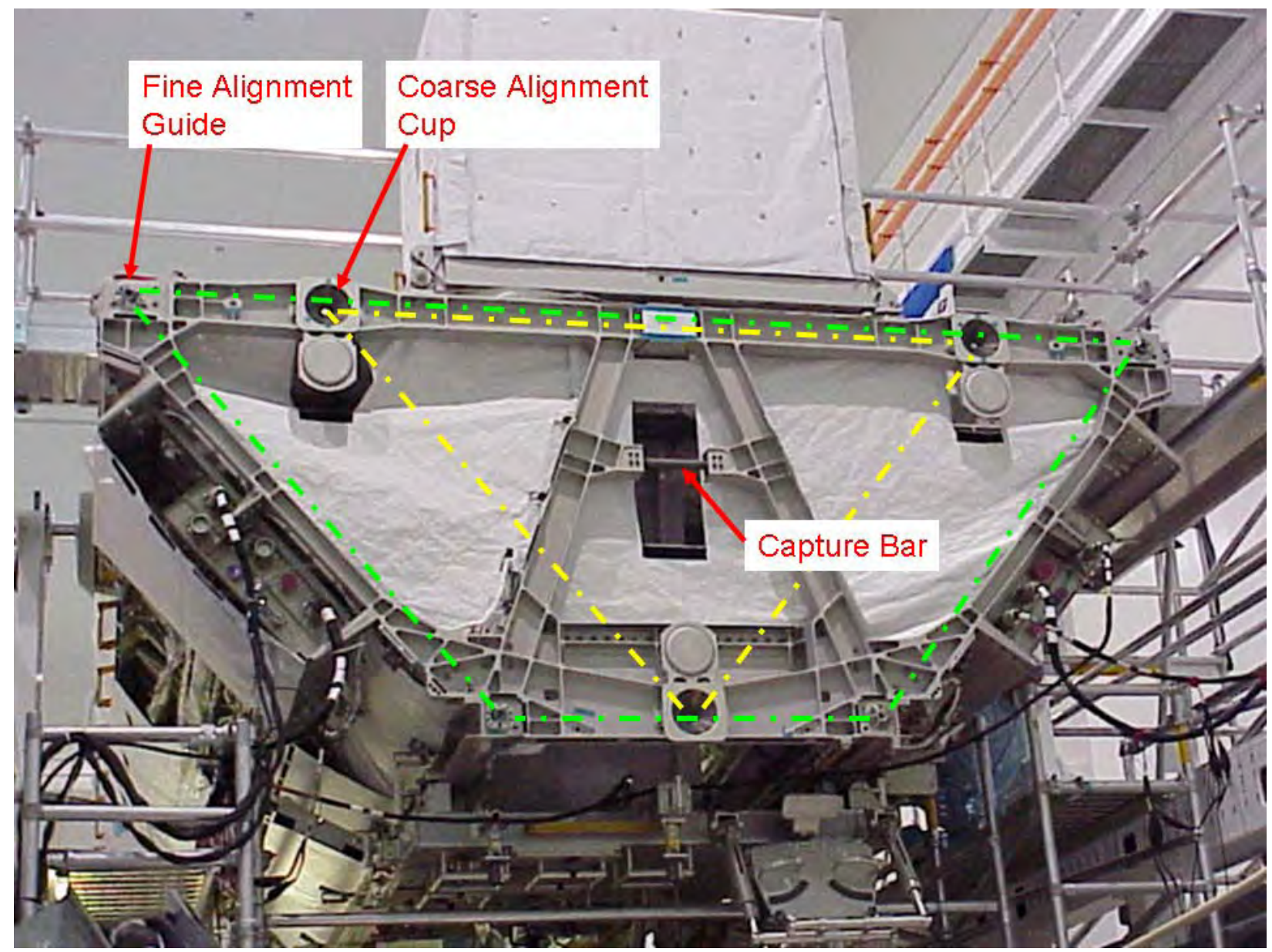

Fig. 23 View showing passive SSAS [25]

\section{Rocketdyne Truss Attachment System (RTAS)}

The RTAS is an attachment mechanism designed by Boeing Heritage Rocketdyne to attach the Z1 to the P6, and later to attach the P6 to P5 and S6 to S5. It consists of four cups and cones for coarse alignment with a capture claw and capture bar with fine alignment bolts as well as contingency fine alignment bolts. When the robotic arm operator places the incoming element where the coarse alignment cones just cross the plane of the coarse alignment cups, the claw is closed, capturing the capture bar and drawing the incoming element down, where coarse alignment features mesh and then the fine alignment features seat. Then the Extra Vehicular Activity (EVA) crew member drives the bolts within the fine alignment features to achieve structural attachment.

The generic features of the Active RTAS are the: 
Coarse Alignment Cup

Fine Alignment Guides

Capture Latch

The generic features of the passive RTAS are the:

Coarse Alignment Cone

Fine Alignment Guides

Capture Bar

A view of the active and passive RTAS is shown in Figures 24 and 25.

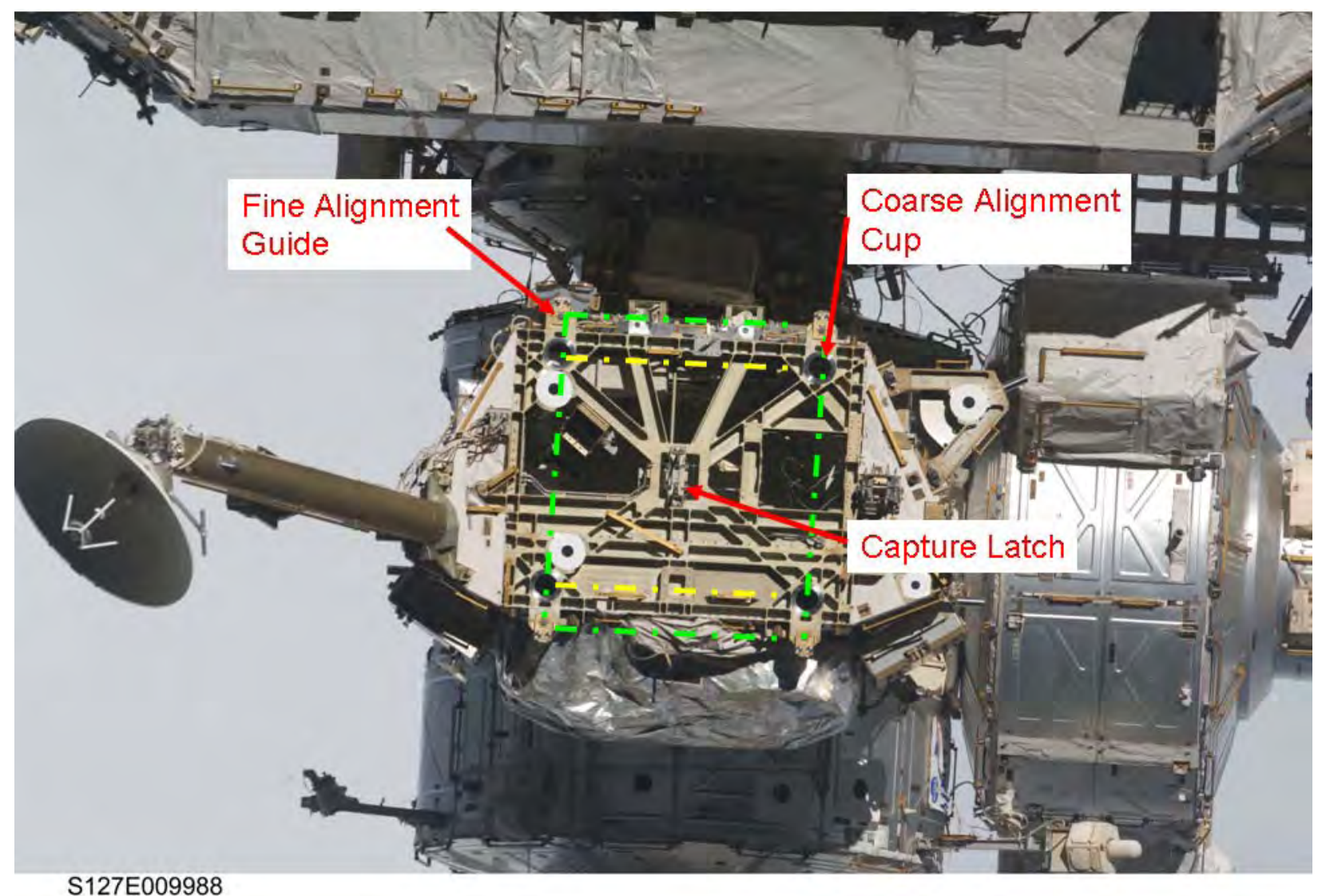

Fig. 24 View showing active RTAS

Image courtesy of NASA [26] 


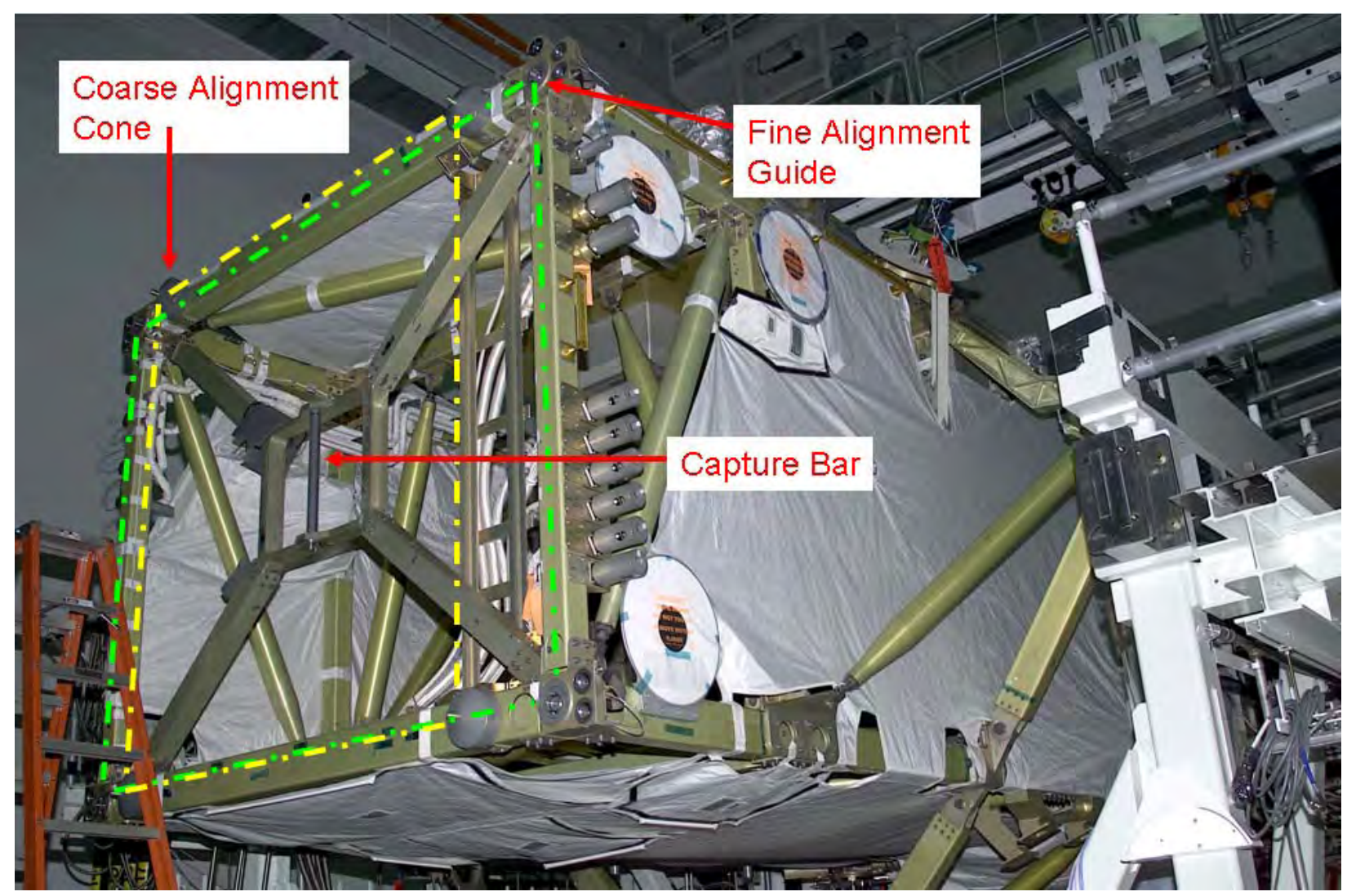

Fig. 25 View showing passive RTAS

Image courtesy of NASA [27]

3. Modified Rocketdyne Truss Attachment System (MRTAS)

The MRTAS is used for the interface for outboard truss segments; between P5 and P4 and S5 and S4. Is consists of four coarse alignment cones, and “stingers” which are in the coarse alignment cups with a spring loaded mechanism that soft captures a slot in each coarse alignment cone when the coarse alignment cone is inserted into the coarse alignment cup. Once all four stingers have been captured, bolts in the fine alignment features (the same found on the RTAS) are seated. Generic features of the active MRTAS are the:

Coarse Alignment Cup

Fine Alignment Guides

Capture Stingers

Generic features of the passive MRTAS are the:

Coarse Alignment Guides

Fine Alignment Guides 
The passive and active MRTAS are shown in Figures 26 and 27.

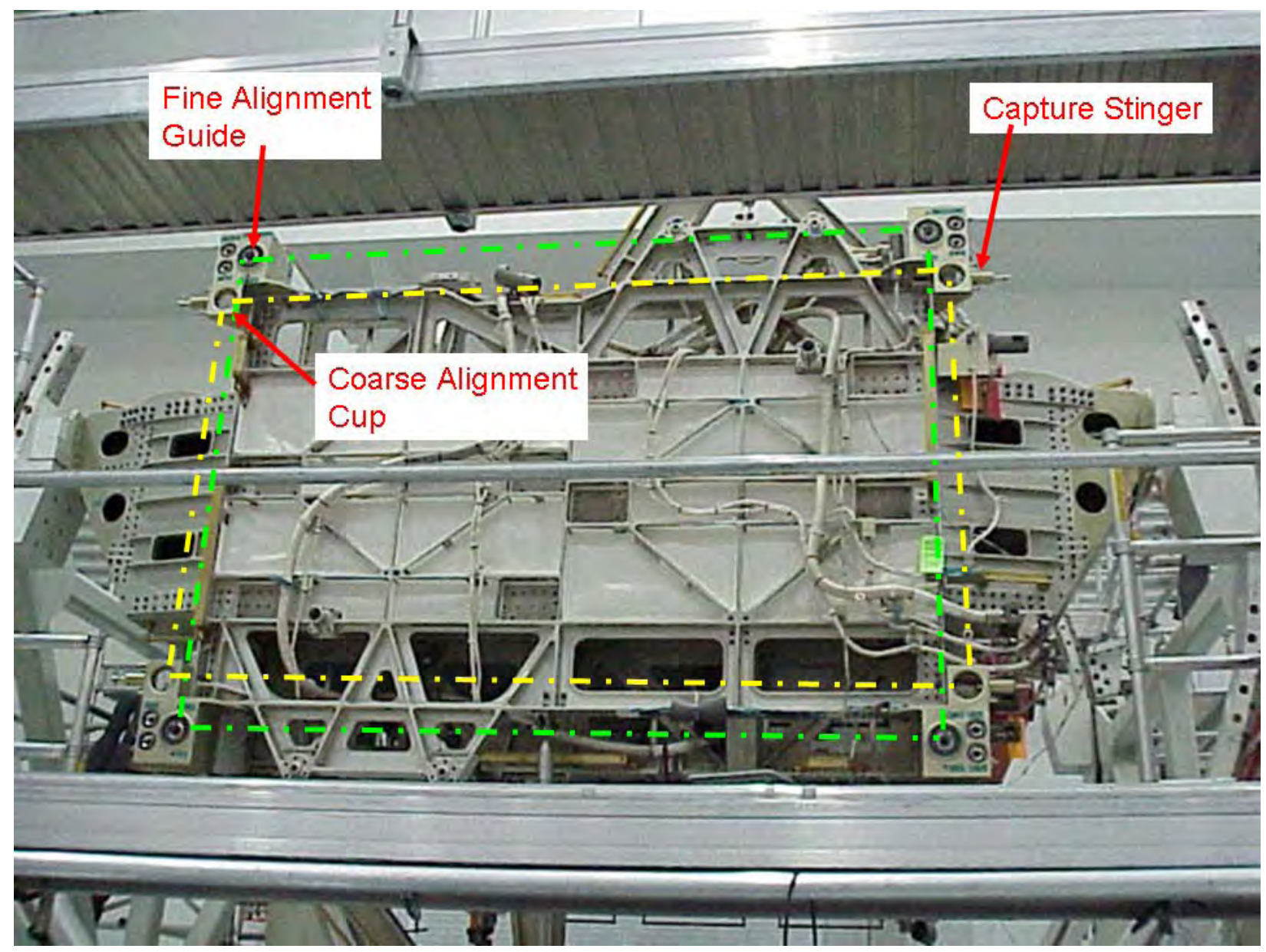

Fig. 26 View showing active MRTAS [28] 


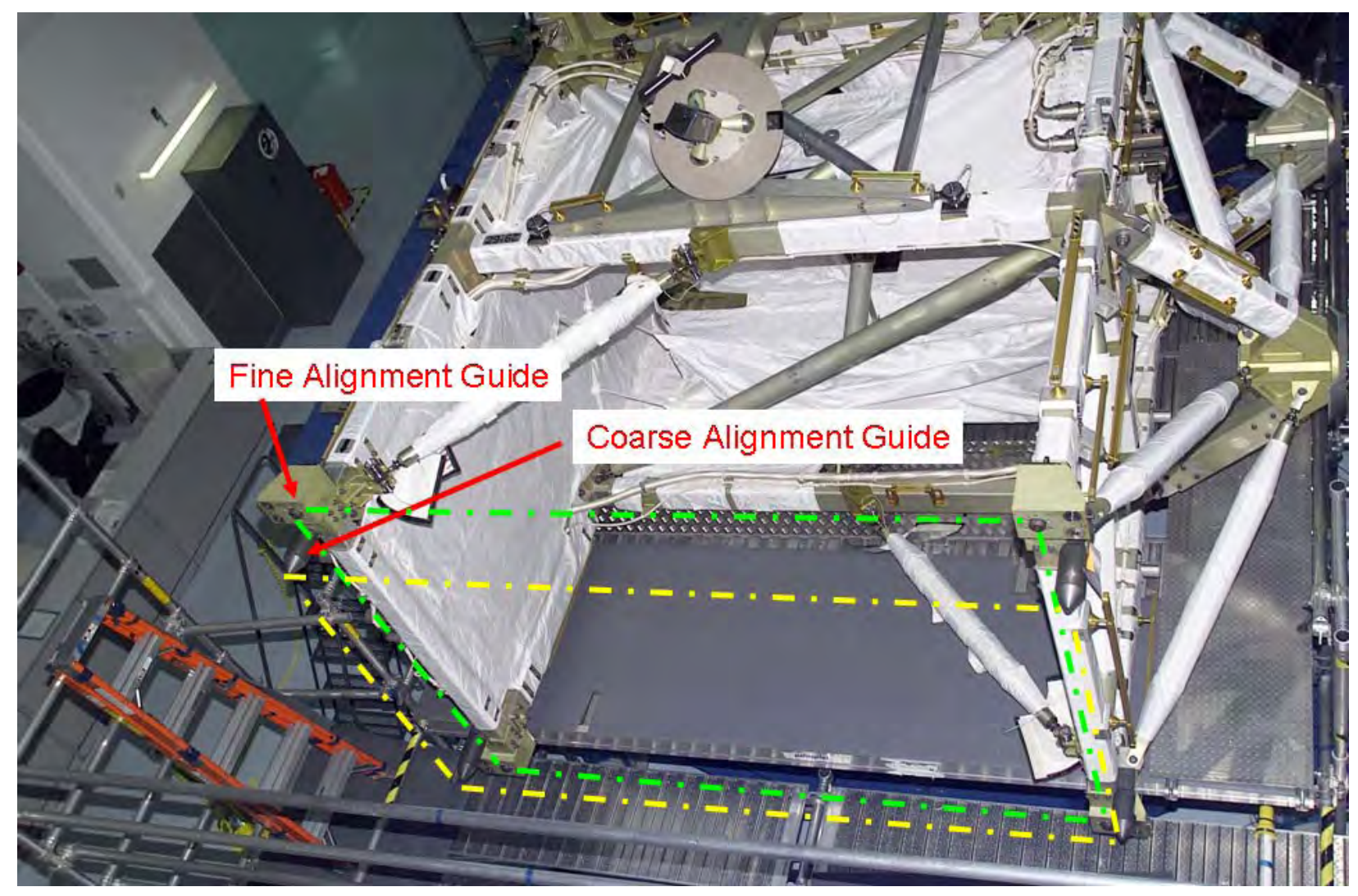

Fig. 27 View showing Passive MRTAS

Image courtesy of NASA [29]

\section{P. Pin and V-guide coarse alignment with central capture/structural latch.}

\section{Module to Truss Segment Attachment System (MTSAS)}

The MTSAS is the interface between the S0 Truss and the Lab. The active half of the interface consists of four V-guides with integrated ready to latch indicators, a capture claw and three fine alignment features. The passive half of the interface consists of four coarse alignment pins, a capture bar and three fine alignment features. When the robotic arm operator brings the incoming S0 passive MTSAS into close proximity with the Lab active MTSAS, the coarse alignment pins cross the "goal posts" of the V-guides and trip the ready to latch indicators. When the proper number of the ready to latch indicators are tripped, the claw is closed, capturing the capture bar. The pins are then seated further in the V-guides until they bottom out in the valley of the V-guide. Bolts in the fine alignment features are driven to structurally attach the mechanisms. Generic features of the active MTSAS are the: 
Coarse Alignment Guides/Ready to Latch Indicators

Fine Alignment Guides

Capture Latch

Generic features of the passive MTSAS are the:

Coarse Alignment Pins

Fine Alignment Guides

Capture Bar

A view of the active and passive MTSAS is shown in Figures 28 and 29.

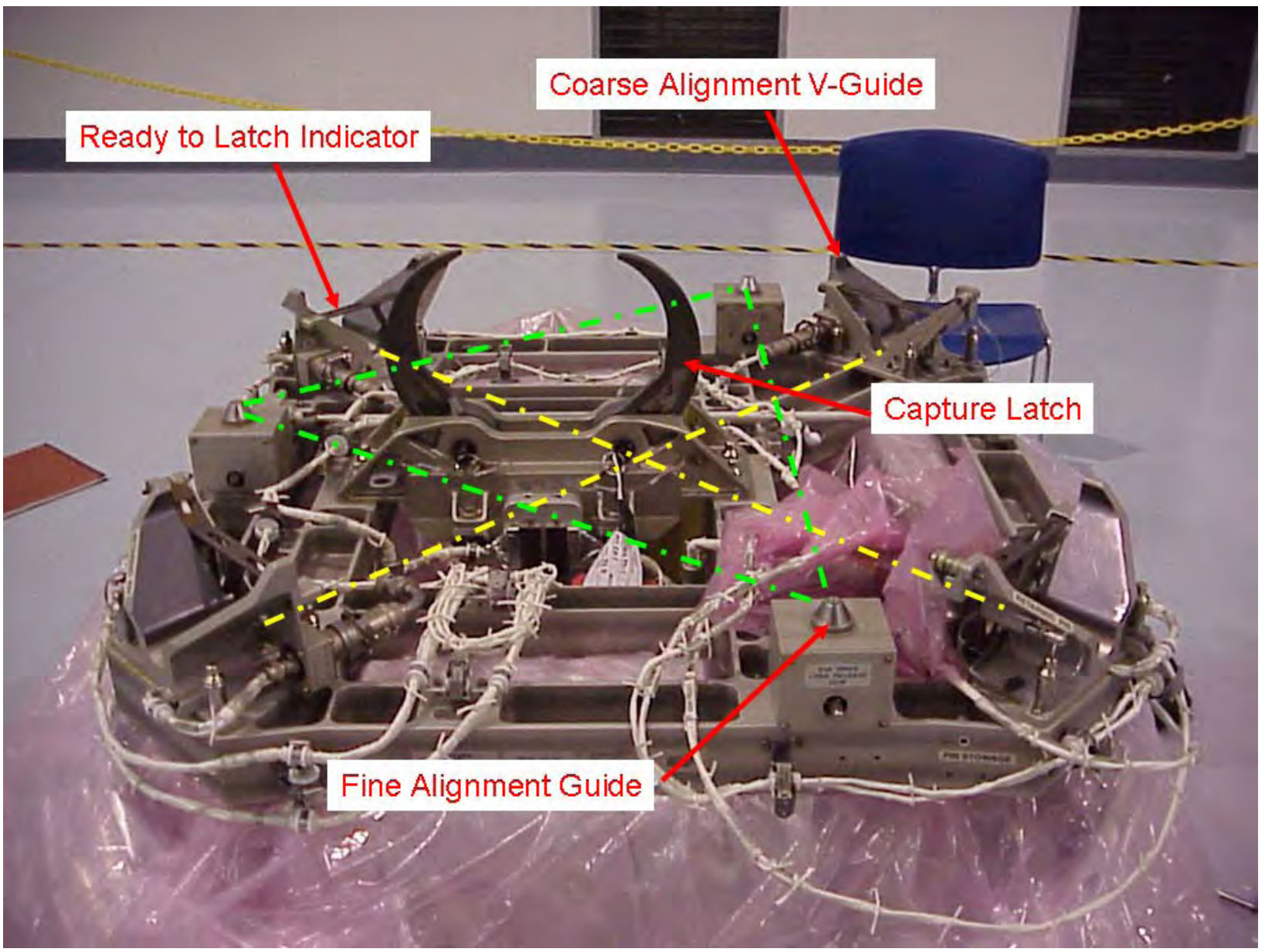

Fig. 28 View showing an Active MTSAS [30] 


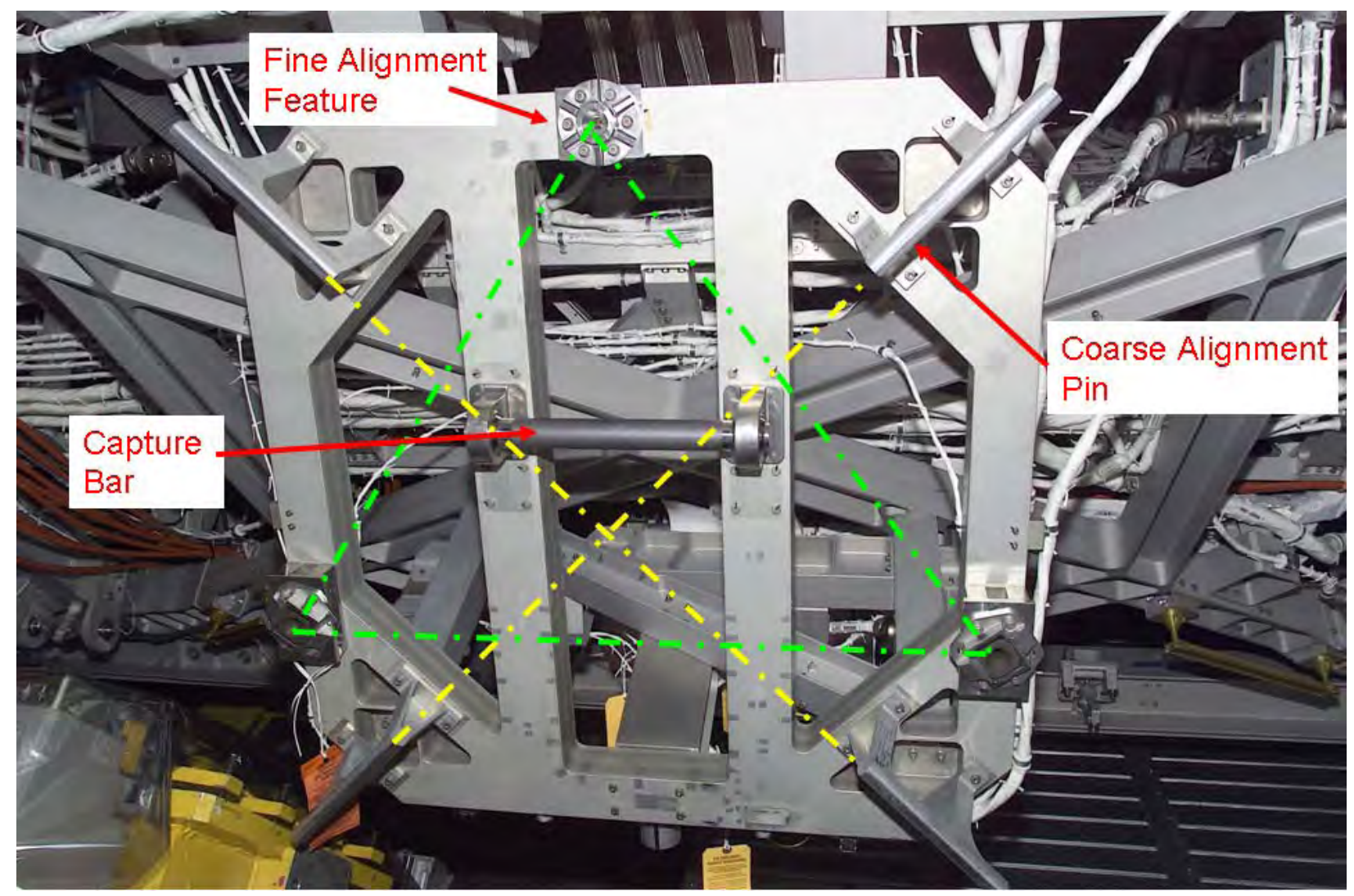

Fig. 29 View showing a passive MTSAS

Image courtesy of NASA [31]

\section{Carrier Attachment System (CAS)}

The stowage of pallets such as the ESP3 and ELCs and the element called AMS use an interface called the CAS. This CAS exists in subtle variations across the station in either truss sites or on the MCAS interface, which is on the MBS on the Mobile Transporter, a small transporter that translates along rails on the Truss face.

The key features of the active CAS are the three V-guides with integrated Ready to Latch (RTL) gate, and a capture claw. The passive half of the mechanism has three coarse alignment guide pins and a capture bar. The robotic arm operator brings the incoming element into close proximity of the CAS site so that the coarse alignment pins cross the "goal posts" of the v-guides and then trip the RTL gates, similar to the MTSAS. The claw is then closed, capturing the bar and seating the coarse alignment guide pins in the channel of the v-guides. Structural attachment is achieved by closing the claw. There are no fine alignment features on the CAS. Generic features of the active CAS are the: 
Coarse Alignment V-guides/Ready to Latch Indicators

\section{Capture Claw}

Generic features of the passive CAS are the:

\section{Coarse Alignment Pins}

Capture Bar.

A view of the Active and Passive CAS are shown in Figures 30 and 31. 


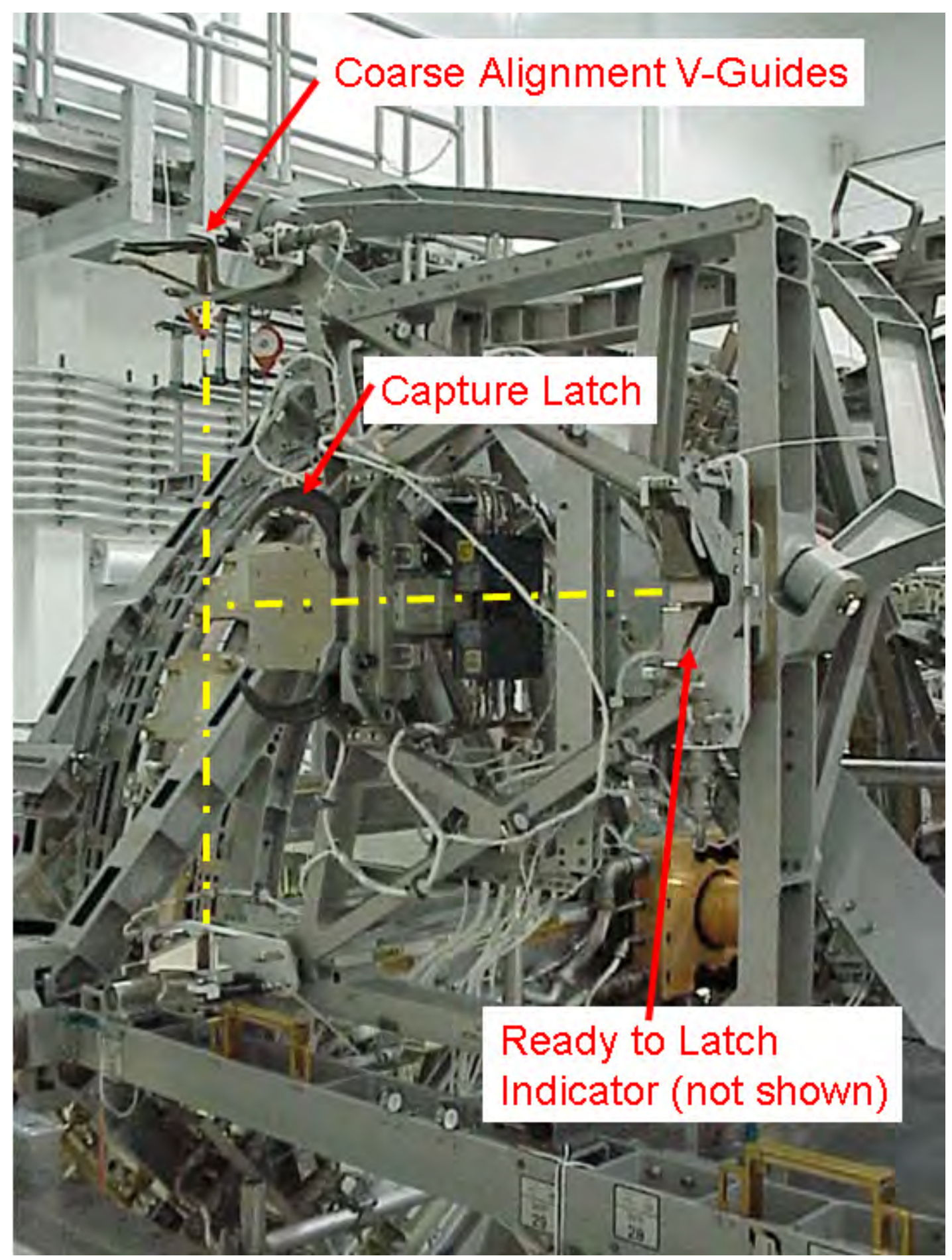

Fig. 30 View showing an active CAS [32] 


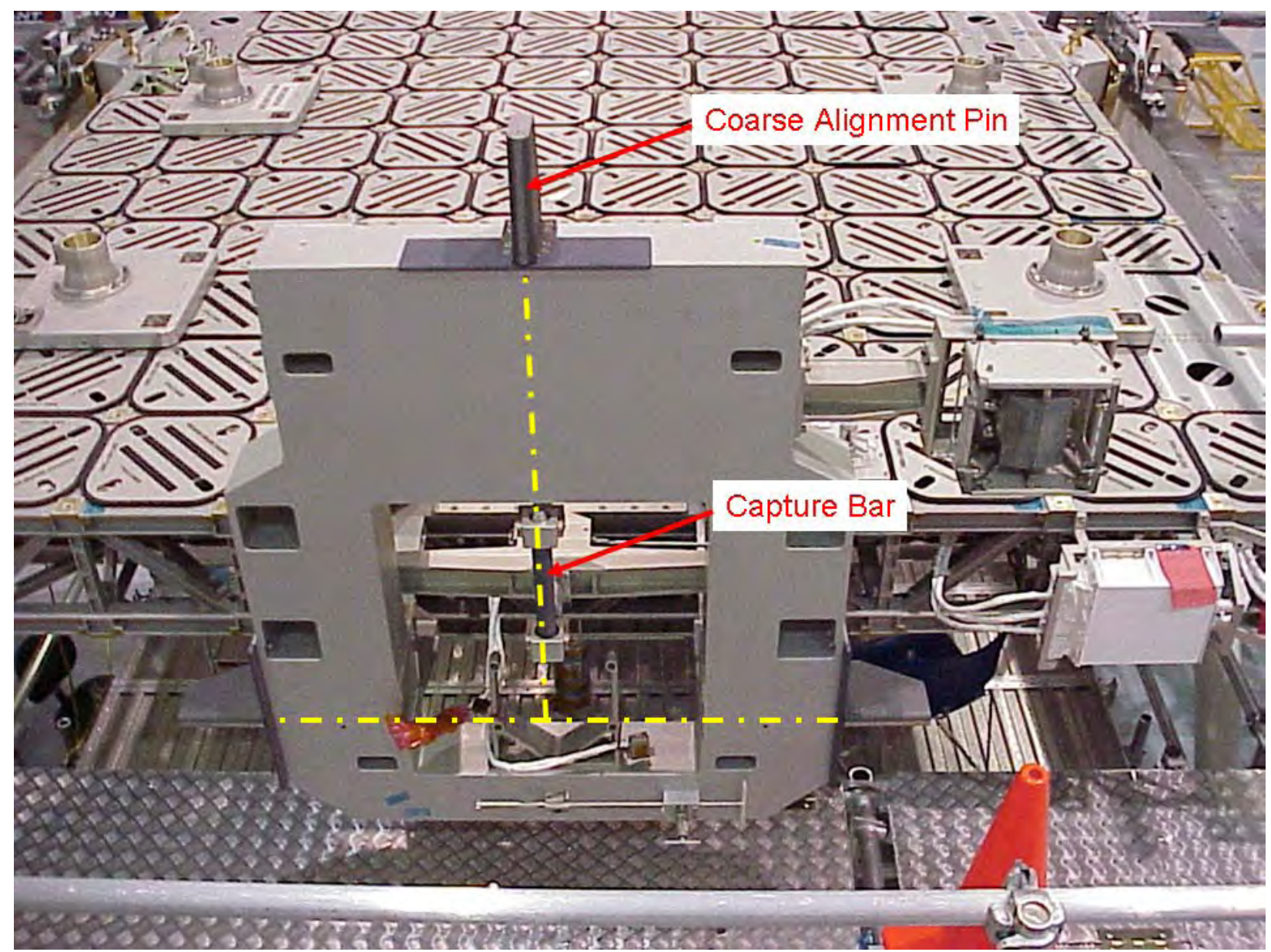

Fig. 31 View showing a Passive CAS [33]

\section{Mobile Transporter (MT) to Mobile Base Servicer (MBS) interface}

The MT to MBS interface is related to the CAS. It has a capture claw, capture bar and pins and V-guides. However the MT to MBS interface has fine alignment features. An unusual aspect of the MT to MBS interface is that the incoming element (the MBS) acts in the active capacity with the capture Claw and V-guides, and the MT side of the interface acts in a passive manner. Usually the capture claw as well as the V-guides are on the ISS side of the interface. The robotic arm operator places the MBS in close proximity of the MT half of the interface and the coarse alignment features interact. When visual cues indicate they are at the ready to latch position, the capture claw captures the capture bar and the four coarse alignment features seat, along with the four fine alignment features. Four bolts then advance to structurally attach the MBS. Generic features of the active half of the MT to MBS interface are the: 
Coarse Alignment Guides

Fine Alignment Guides

Capture Latch

Generic features of the passive MT to MBS interface are the

Coarse Alignment Pins

Capture Bar

A view of the active and passive half of the MT to MBS interface is shown in Figures 32 and 33.

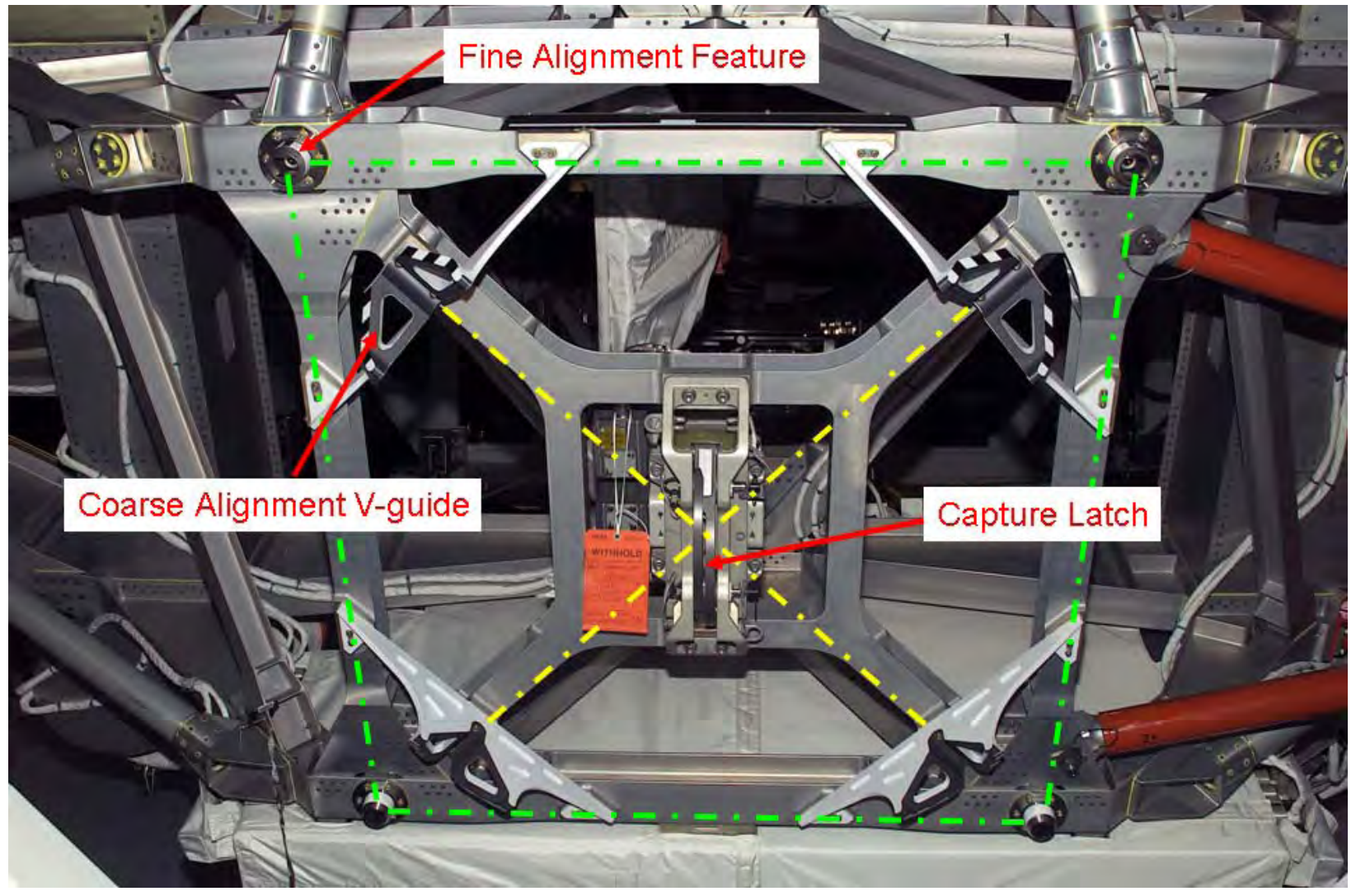

Fig. 32 View showing the active half of the MBS to MT interface Image courtesy of NASA [34] 


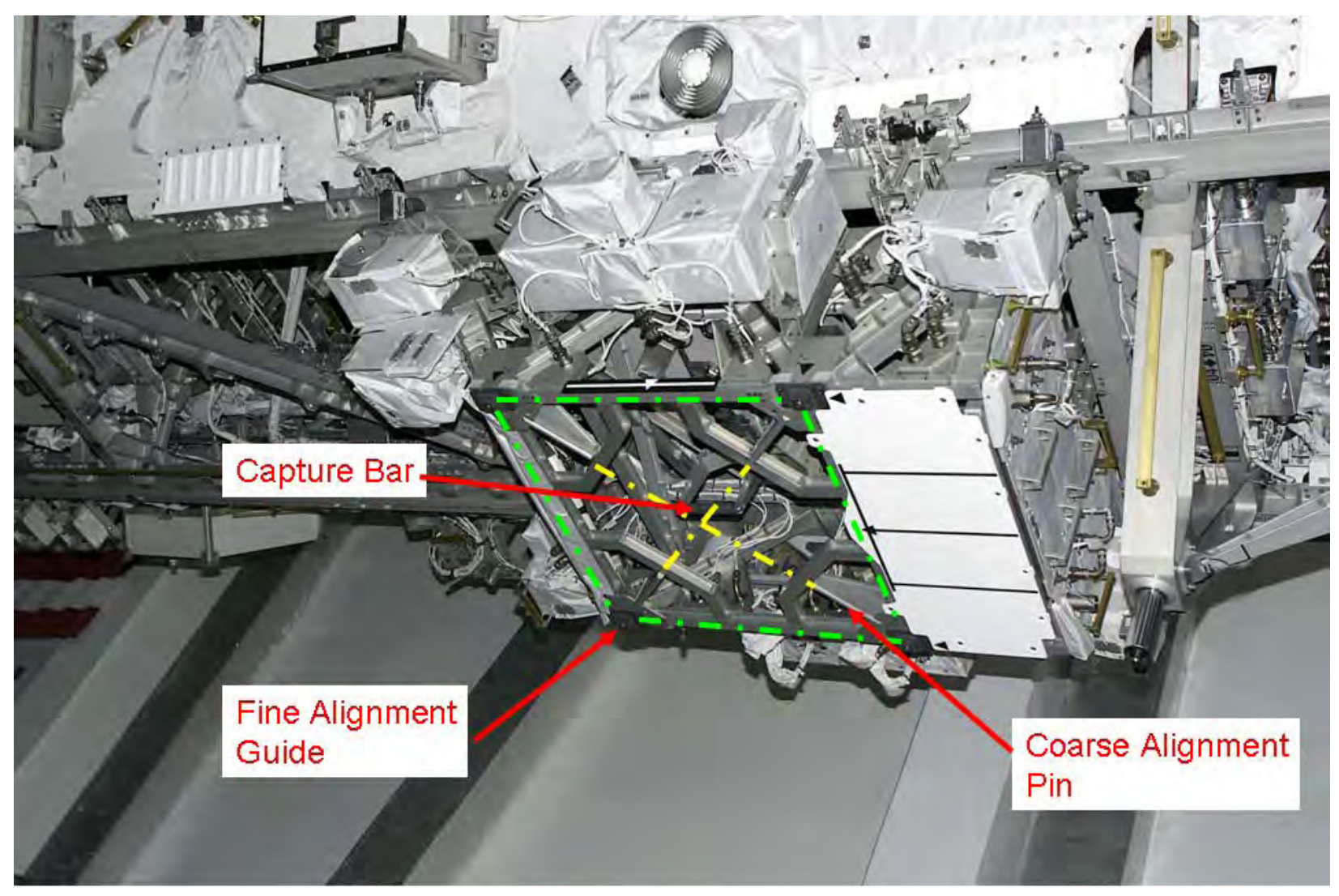

Fig. 33 View showing the MT side of the MT to MBS interface

Image courtesy of NASA [35]

\section{Q. Active and Passive with latches and bolts}

\section{Manual Berthing Mechanism (MBM)}

The need for a temporary berthing location for PMA3 during 5A was resolved by the use of a Boeing Manual Berthing Mechanism. This is similar to an Active Common Berthing Mechanism, except that the mechanisms are manually operated instead of electrically operated. Figure 34 shows the MBM 


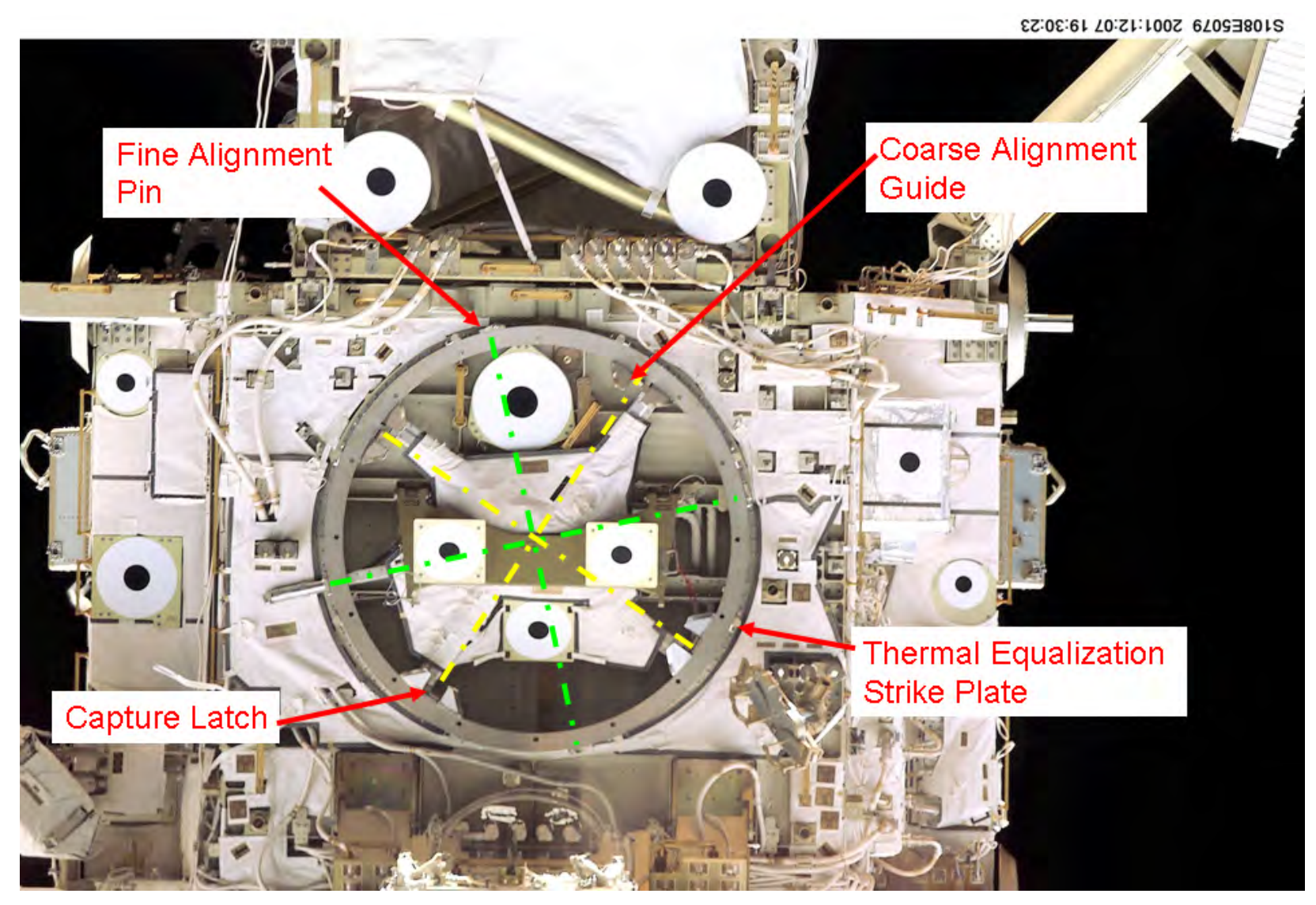

Fig. 34 View showing the MBM

Image courtesy of NASA [36]

2. Exposed Facility Berthing Mechanism (EFBM)

The Exposed Facility Berthing Mechanism (EFBM) is used to berth the JEM EF and the JEM PM modules. The EFBM is made up of two mating halves; an Active and a Passive half. The generic features of the EFBM active half are:

Coarse Alignment Guides

Alignment Sockets

Capture Latches

The generic features of the EFBM passive half are:

Coarse alignment guides

Alignment Pins 
When the passive EFBM is brought into proper alignment with the active EFBM, the coarse alignment guides provide coarse alignment by constraining roll and translation. As the incoming passive EFBM is translated towards the active EFBM, the coarse alignment guides interact with each other as needed. When EVA crewmembers determine the mechanism is ready to latch, the capture latches are deployed and draw the EFBM into fine alignment. This is achieved when the EFBM fine alignment pins are seated in the EFBM fine alignment sockets. Once aligned, structural attachment is achieved by fully retracting the capture latches. A view of the active half and passive half of the mechanisms is shown in Figures 35 and 36.

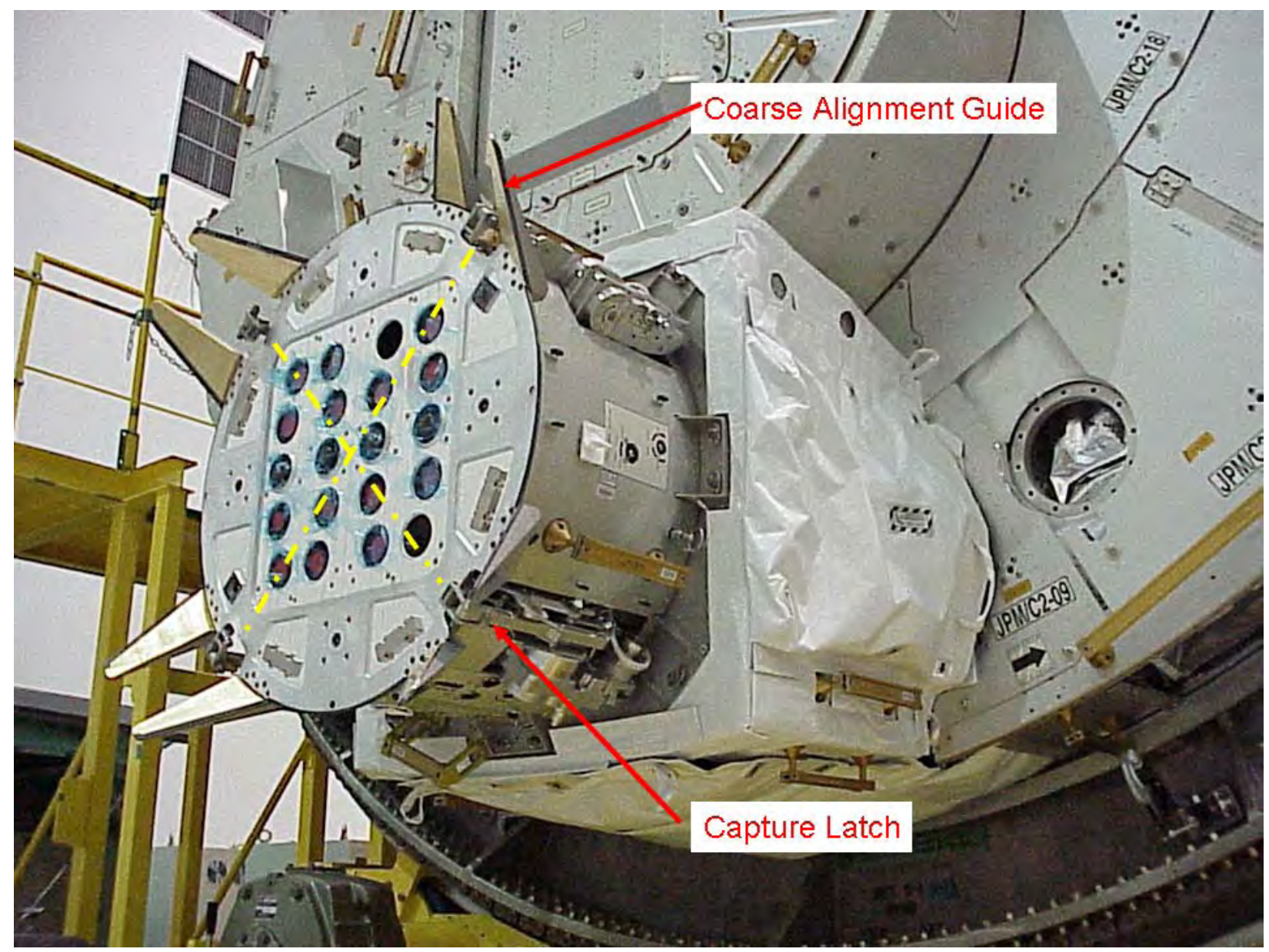

Fig. 35 View showing active EFBM [37] 


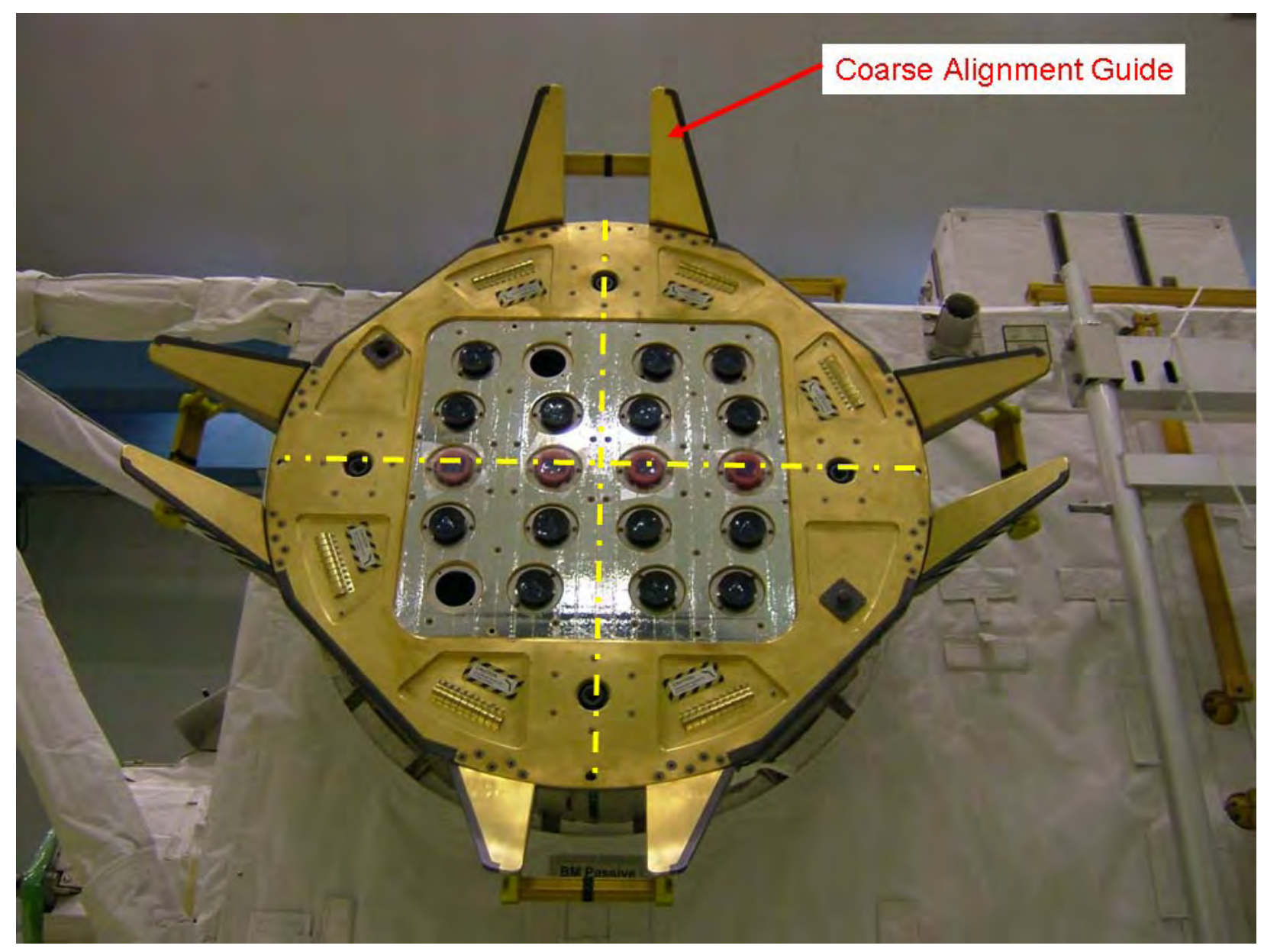

Fig. 36 View showing passive EFBM [38]

\section{Exposed Facility Unit (EFU)}

The Exposed Facility Unit (EFU) is used to berth between the JEM ES and the JEM EF modules. The EFU is made up of two mating halves; an Active (EFU) and a Passive half Payload Interface Unit (PIU). The generic features of the EFU active half are:

Capture Latch Arms

Alignment Sockets

The generic features of the PIU passive half are:

Coarse alignment V-guide

Alignment Pins

Berthing Plate (protective cover) 


\section{Damper (central plunger)}

When the passive EFU is brought into proper alignment with the active EFU, the Capture Latch arms are closed, and they seat in the V-guides. Interaction with the V-guides aligns the PIU with the EFU. The capture latches continue to pull in and draw the PIU central damper in contact with the EFU protective cover. As the damper is compressed, the PIU Berthing Plate is compressed. Further compression brings the PIU into fine alignment. During fine alignment, the PIU alignment pins compress to seat in the EFU fine alignment sockets. Once aligned, structural attachment is achieved by fully retracting the capture latches. A view of the active and passive EFU are shown in Figures 37 and 38.

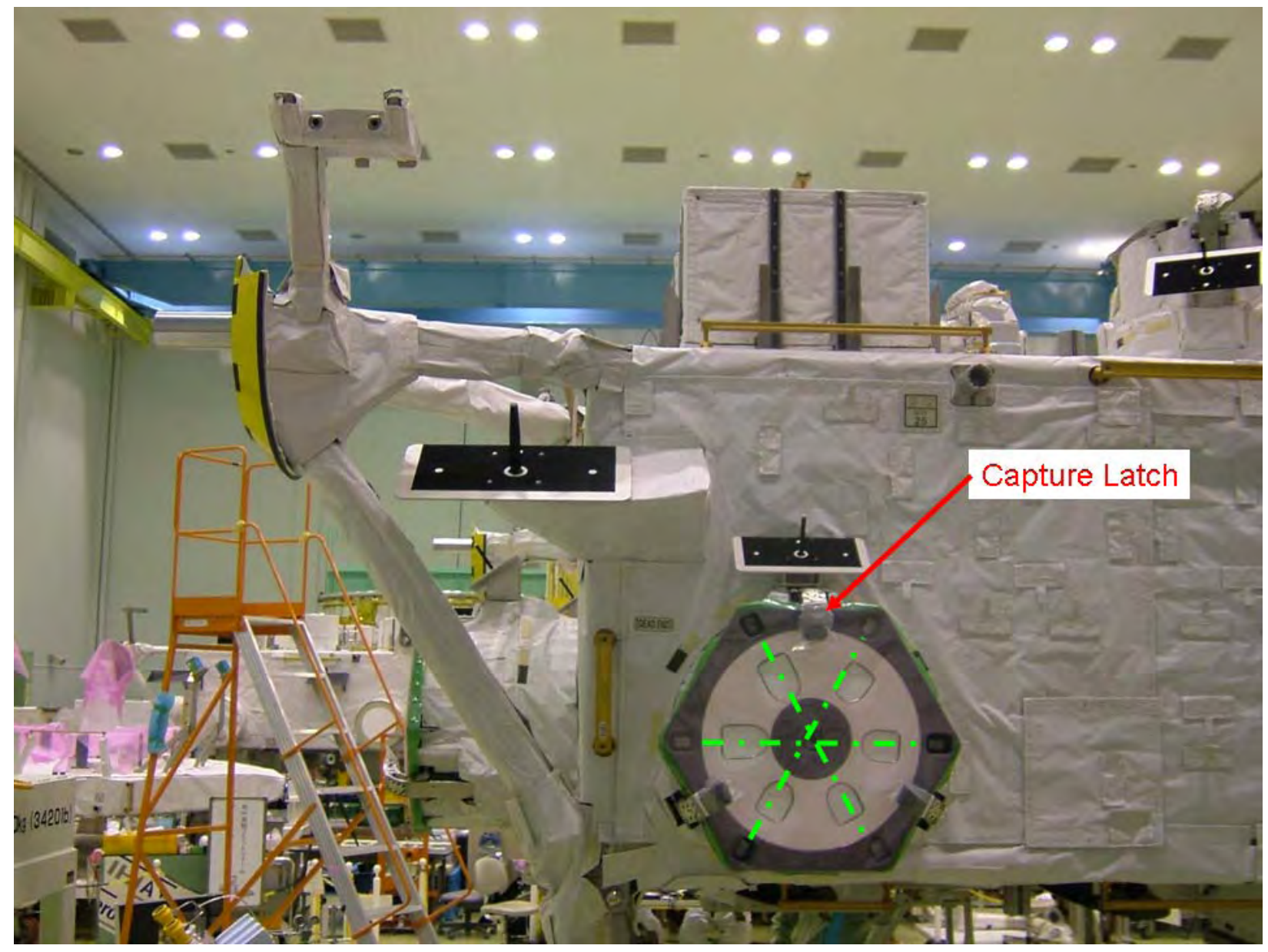

Fig. 37 View showing EFU [39] 


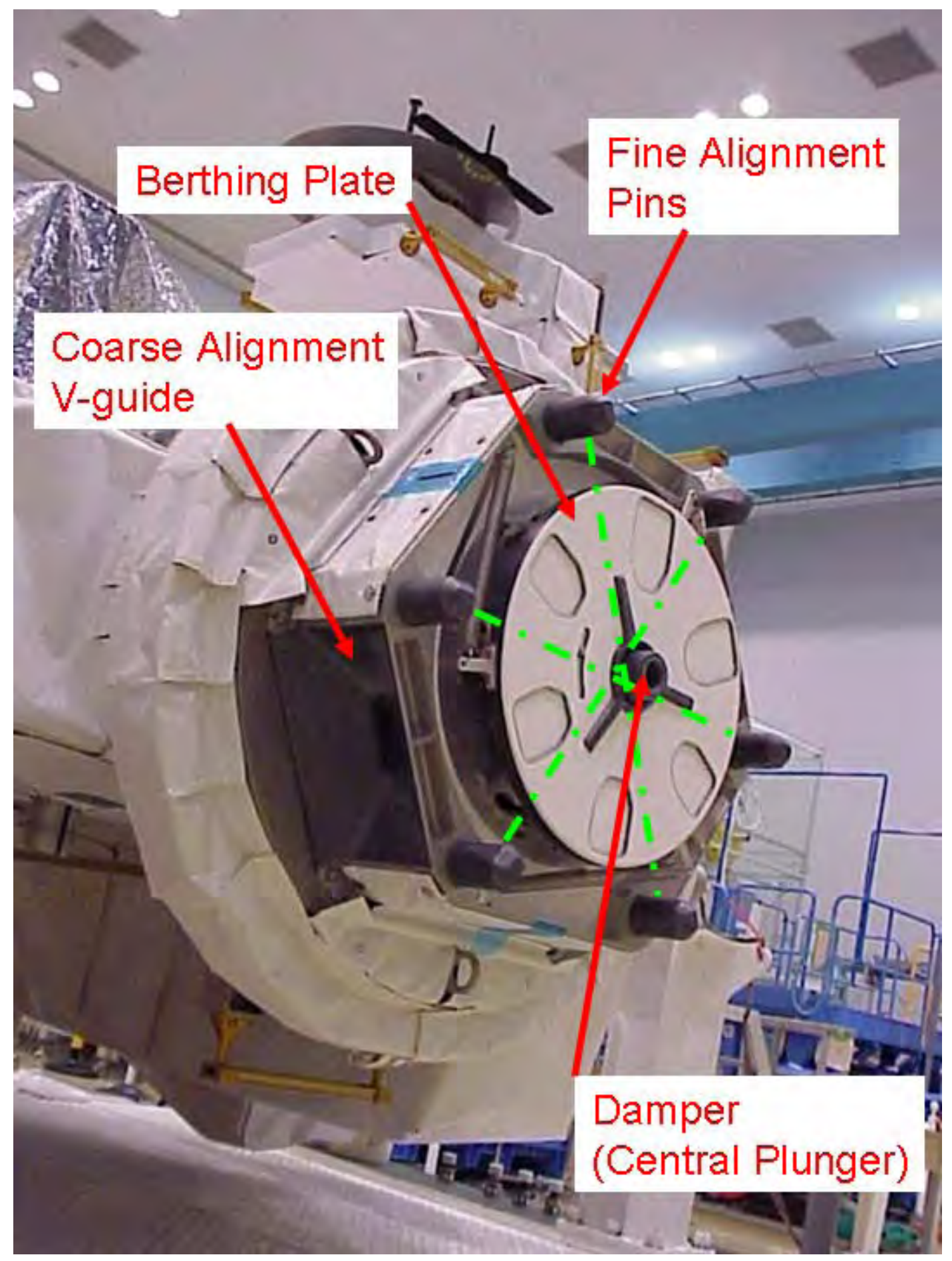

Fig. 38 View showing passive EFU [40] 


\section{Next Generation}

\section{A. LIDS}

NASA's Low Impact Docking System (LIDS) has been in development since the mid-1990s. Originally undertaken in support of the X-38 program which was an ISS crew return vehicle prototype, the LIDS offered a magnetic soft capture system coupled with a computer controlled load attenuation system which could potentially greatly reduce the initial docking loads required to effect soft capture between mating vehicles in space.

"All previous docking mechanisms have required the use of impacts (i.e. velocity or post-contact thrusting) to create the energy required for soft capture mechanism interface alignment and capture between mating docking interfaces. Low Impact technology can be used to greatly reduce and even eliminate the need for impact energy and provide the flexibility for future mission planners” [41].

Like APAS, the LIDS can also trace its origins back to the Apollo Soyuz Test Project docking mechanism. After the ASTP came the McDonnell Douglas Space Station Docking Mechanism which was developed for Shuttle to Space Station Docking in the mid 1980s. It also used peripherally mounted alignment and capture mechanisms with computer controlled electromechanical actuators for the load attenuation system. This system was ground tested but never flew as the program eventually elected to use the Russian APAS for this task.

The LIDS soft capture system uses a series of magnets which, in concert with their associated strikers (contact surfaces), are capable of significantly reducing impact loads typically associated with soft capture. The LIDS soft capture ring integrates a load sensing ring which senses loads and, using a complex control algorithm, dampens oscillations and maintains horizontal alignment between the mated vehicles. The dimensions of the LIDS with magnets and load sensors installed results in an inside diameter (passageway diameter) which is different than APAS. The outer diameter for the two soft capture rings is the same for both systems; however the inner passageway diameter resulting from the different arrangements of petals used on LIDS as opposed to APAS is quite different. The passageway diameter inside the APAS docking ring is $800 \mathrm{~mm}$. The LIDS soft capture ring with petals in place provides a passageway diameter of $685 \mathrm{~mm}$. However, there is the potential for having the capability to remove the petals on the LIDS soft capture ring. Removing the petals on the LIDS creates a passageway diameter equivalent to the APAS diameter of $800 \mathrm{~mm}$. A view of the LIDs docking mechanism is shown in Figure 39. 


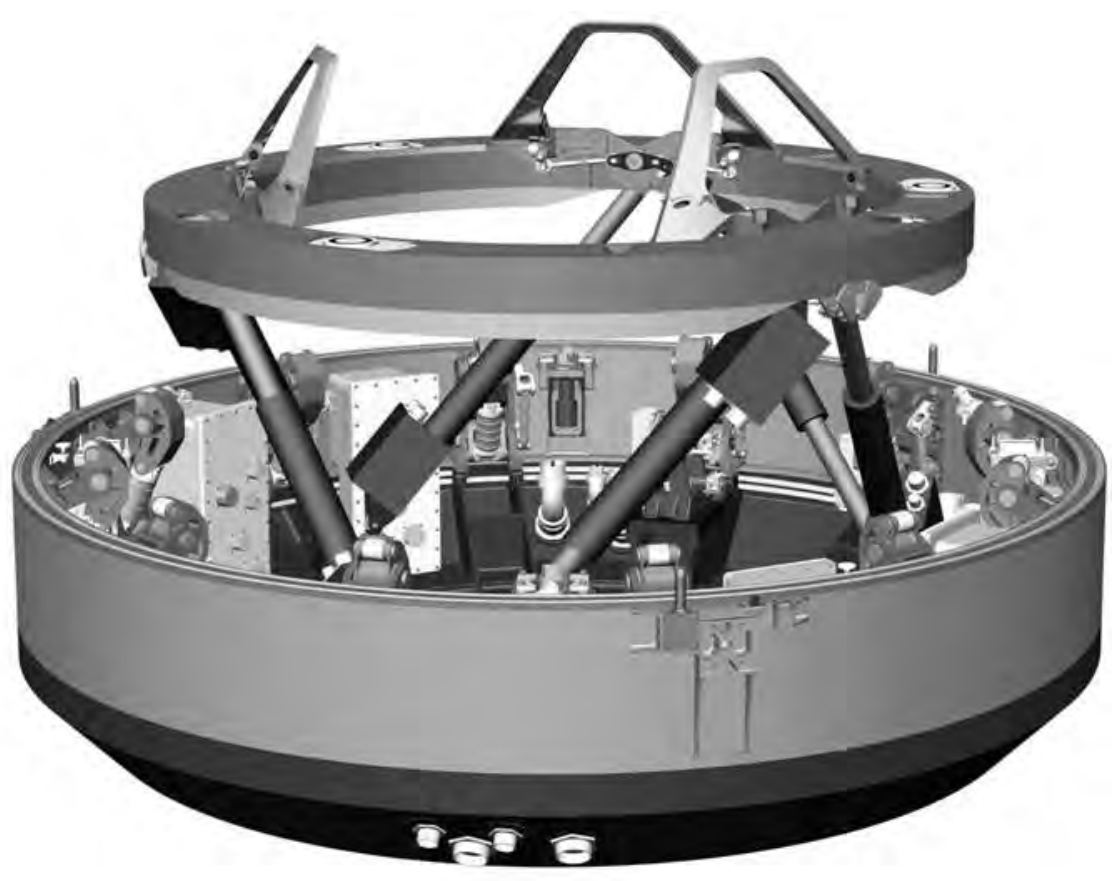

Fig. 39 View showing LIDS in Active Mode Image courtesy of NASA [41]

\section{B. IDSS}

In the 2008 timeframe NASA began negotiations with the ISS International Partners (Roskosmos, ESA, CSA, and JAXA) on the development of a new International Docking System Standard (IDSS). It was envisioned that a common mechanism design would enable joint operations between countries in the future, both in low earth orbit and exploration missions to the moon or beyond. NASA proposed its LIDS mechanism be considered as the starting point for a standard. By 2010 negotiations yielded a compromise; the hard capture system would be based on the APAS and the soft capture system would be based on the low impact LIDS technology.

When the last Space Shuttle undocks from the International Space Station it will be the last time that a space vehicle will dock to the APAS system at that location. NASA has endeavored to develop a brand new system for docking to the International Space Station. NASA and its partners on the International Space Station; including European, Canadian, Japanese, and Russian Space Agencies, have been collaborating on development of an international standard for docking which would enable on-orbit crew rescue operations and joint collaborative 
endeavors utilizing different spacecraft. The result of this collaboration is the introduction of the International Docking System Standard Interface Definition Document (IDSS IDD). It provides a detail description of the physical geometric interface requirements necessary in achieving a physical connection between two vehicles. It also has a comprehensive description of performance parameters required including initial conditions for capture (lateral, angular and rotational misalignments on approach and velocity), vehicle mass properties, and dynamic loading conditions. The IDSS IDD does not specify an implementation of these interface requirements. Rather, it provides enough information for spacecraft developing entities to independently perform all of the design, analysis, and certification necessary to build a system capable of mating with the docking interface. The interface represented in IDSS for the soft capture reflects that of the Low Impact Docking System used on NASA's Docking System (NDS). The soft capture system used on APAS uses a narrower ring width dimension which places the three alignment petals at a larger diameter than the petals on LIDS. As designed, the APAS soft capture ring is not compatible with the IDSS standard interface. The hard capture interface that has been integrated into the IDSS is almost entirely based off of the Russian APAS system although significant differences exist including the retractable pusher mechanism, sensor suite, retractable electrical connectors, and pyrotechnic separation system. Dimensional interfaces for the structural body, hooks, and pressure seal diameters are the same as is used on APAS. As of this writing, international agreement on this arrangement in the IDSS IDD has been reached with three of the four partners. The Russian Space Agency, Roscosmos, is still in negotiations for providing approval to the IDSS standard interface document.

\section{NDS}

In 2010 the LIDS requirements were updated to include the new IDSS standards, which led to a redesign of the hard capture system. The new system was dubbed the International Low Impact Docking System or iLIDS and is also known as the NASA Docking System (NDS). Currently the NDS is on track to become the first docking mechanism built to the IDSS. The NDS Critical Design Review (CDR) was recently completed in early 2011 and plans call for completing the flight certification by 2014. The NDS is shown in Figure 40. 


\section{Ready to Dock - Active Mode (All Configurations Except as Noted)}

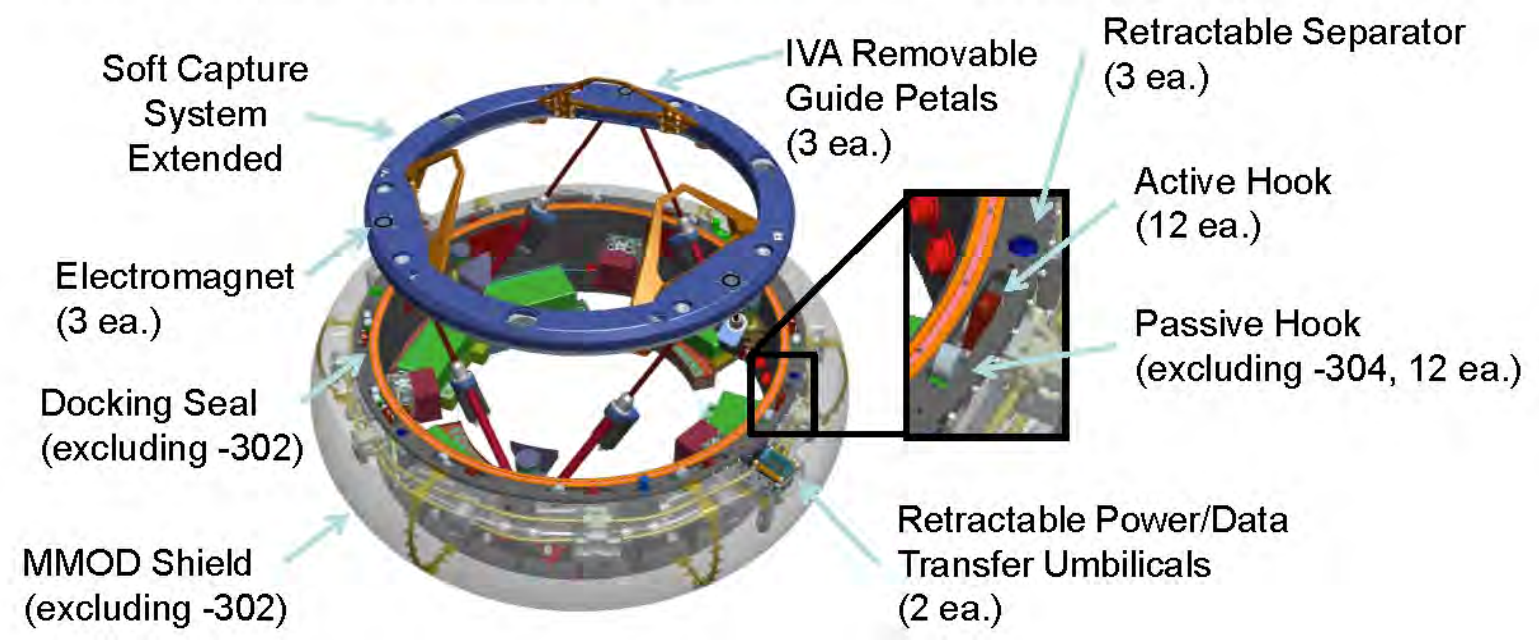

Fig. 40 View showing NDS in Active Mode

Image courtesy of NASA [42]

\section{APAS with modified avionics}

As part of the plans for developing a commercially based domestic crew launch system Boeing has considered the Russian designed APAS system as an option for providing docking capability. The APAS system has flown in its two main configurations on Space Shuttle to both the MIR and ISS stations. Boeing determined that one of the main areas of improvement in the APAS system would be to pursue a modernization and consolidation of the existing Russian avionics. The existing APAS system flying on the Space Shuttle consists of nine avionics instruments. They provide power distribution, control, data collection, and incorporation of back up systems. This results in a large footprint for installation and the many cables required result in a considerable amount of weight. An internal research and development project was initiated in parallel with Boeing's involvement on Commercial Crew Development (CCDev) project. The existing APAS avionics which uses an extensive array of electronics relays and switches would be replaced by a more modern microprocessor or logic based system using fewer components, lower power consumption, increased fault detection capability, smaller footprint and volume for installation, and reduced weight. 


\section{Future Uses}

\section{A. ISS Docking Hub}

A docking hub would be an excellent addition to the ISS. It would allow for additional expansion to add to the space station and to allow for the use of the ISS as a transportation hub for future exploration. Boeing is leading an effort to design a docking hub. The docking hub would be fabricated from a Node 1 structural test article. Likely interface mechanisms for such a hub would be NDSs and CBMs.

\section{B. Commercial Crew Transport}

With the shuttle being retired, there is an urgent need for crew transport to the ISS. Reliance on foreign means for transporting crew and cargo to the US segment is both costly and detrimental to the future of the American space program. As such, NASA has decided that the future of transporting both crew and cargo, to and from the ISS, is to be made available from the commercial space industry. Numerous vendors are working on a crew transport and Boeing is developing the CST-100 to transport crews to ISS.

Boeing has participated in the NASA requests for proposal to develop a commercially produced domestic crew launch and return capsule capable of reaching Low Earth Orbit. The Boeing capsule design has been pursued as part of the Commercial Orbital Transportation Services (COTS) proposal to develop the capability for transporting crew and cargo to and from the ISS. Boeing's most recent involvement in the competition for providing a commercial system has been under the Commercial Crew Development project, CCDev. It is the next phase in the development of commercial crew spaceflight concepts and associated technologies. Initial development of the Boeing crew capsule was to include the APAS as the docking system for attaching to ISS. The Russian APAS is a highly successful system and has been proven extremely reliable over the years. The Boeing team provides an in depth knowledge of the APAS system on Space Shuttle. Their experience using the Russian designed system provided them with many lessons learned. The proposal to utilize the APAS on COTS and CCDev included options for weight reduction, modified dampening systems, and to pursue a consolidation and modernization of the docking system control avionics installations. The landscape changed and the plan for integration of the APAS was

overcome by NASA's desire to implement the International Docking System Standard (IDSS) interface. The current configuration of the APAS is not per the IDSS standard. NASA is in development of the NASA Docking System (NDS) which is NASA's implementation of the IDSS standard docking interface. In order to be able to 
dock with ISS Boeing has made the decision to pursue the NDS docking system. The implementation of the NDS includes a change in electrical and avionics system architecture in order for the vehicle power and controls systems to be able to command and control the Low Impact Docking System (LIDS) soft capture installation. The LIDS utilizes a set of magnets and load sensors to achieve soft capture and to dampen motion prior to achieving hard mate, whereas the APAS uses mechanical latches and spring dampening to do the same. The avionics and power requirements for NDS/LIDS are quite unique as compared to the APAS. Having developed the structure and avionics to accommodate the APAS provides Boeing with the option for docking to an orbital space complex that has either system as its portal of entry.

\section{Commercial ISS resupply}

With the shuttle being retired, there is a need for cargo transport to ISS. The Space Shuttle with its enormous payload bay and its ability to transport up to seven passengers was a very accomplished vehicle for satisfying that function and its versatile capabilities will be hard to replace. Numerous vendors are working on developing cargo transport and some commercial vendors are using CBMs as their interfaces for attaching to ISS and enabling transfer of commercial cargo. Boeing has provided vehicle concepts as part of proposals to NASA on the Commercial Resupply System (CRS) and the Commercial Orbital Transportation Services projects.

\section{Commercial Space Station}

Expandable systems have been in development with NASA dating back to before the 1960s. NASA even considered using expandable systems in the 1980's as part of future planetary expeditions. Bigelow Aerospace has been in development of expandable space habitats since 1999. Most recently, Bigelow has launched two expandable structures to low earth orbit, Genesis I and Genesis II, which were launched in 2006 and 2007 respectively. Bigelow is currently planning to fly an inflatable to ISS. Bigelow is also planning a commercial space station for tourism which will be ready for deployment in 2014, and official habitation by its customers in 2015. Boeing is partnering with Bigelow on the CCDev project. As part of the collaboration Boeing will provide the crew transportation on their commercial crew capsule system to the Bigelow Station. The mechanism to be used for the interface between the CST-100 and the Bigelow module was originally an APAS, but the NDS platform is also consideration due to its implementation as part of the International Docking System Standard (IDSS) 
Bigelow has considered use of the APAS system as the means for attaching the inflatable modules together (inter-module attachment). They have also considered use of the common berthing mechanism. If the choice is made to use the APAS then this would involve a potential use of the new development avionics system for control of the APAS system which Boeing is currently developing.

\section{Future Exploration}

\section{A. Lunar}

The moon remains a relatively near destination that holds great interest to the scientific community and has much potential for exploration and development. The Apollo missions just scratched the surface of what there is to learn about the moon. The resources on the moon in the form of minerals and elements such as hydrogen and oxygen hold much potential for future exploration for consumables and propellant. A CBM would accommodate the current hatch sizes for a lunar habitat, but the CBM was designed for the pristine environment of a vacuum and a redesign would be necessary for the seals and bolts to be able to work in the dusty environment of the lunar surface. Also of consideration are aligning the modules and mechanisms on a potentially uneven lunar surface. Numerous unpressurized interfaces may be applicable for lunar support construction.

\section{B. NEO}

Asteroid impacts have played a prominent role in the development of the solar system and in the active, dynamic geological life of this planet. It is likely that there was a mass extinction caused by an asteroid impact near what is now the Yucatan Peninsula. The threat of asteroid impact is one worth preparing to learn how to avert. Missions to nearby asteroids might allow us to learn more about them and plan how to divert an asteroid's path if it were headed for a collision with Earth. A vehicle that rendezvous with an asteroid will likely have an NDS on it.

\section{Mars}

Mars figures prominently as a likely planet to search for other life in the solar system. Even if there is no life found on Mars, it has much to offer in the potential to terraform a second "Earth". Although such an endeavor would likely take a long period of time, it is worth considering since mankind needs new territory to expand out into the solar system and Mars has a great deal of potential as a base for a colony. Just as on the lunar surface, the 
dusty environment of Mars would pose a challenge for a pressurized attachment mechanism. The same sort of alignment issues would exist as on the lunar surface.

\section{Conclusion}

The first docking mechanisms, the Probe \& Drogue and the Gemini Cone \& Cup interface did not allow transfer of people between vehicles, only docking two vehicles. With the modification of the Probe \& Drogue to allow for the Probe \& Drogue to be rotated out of the way to create a passageway, crew could transfer between vehicles. Likewise with the Apollo Probe \& Drogue, the probe and cone mechanisms could be removed to allow for crew transfer. The Apollo/Soyuz APAS75 had outward facing coarse alignment petals, which freed up space in the crew passageway. Later, for the International Space Station, there was a need for not only crew to transfer between modules, but for the transfer of racks between modules. The size of these racks necessitated a 50" square hatch to accommodate this transfer. Thus the CBM was sized to accommodate this hatch. The new NDS will have removable coarse alignment guides to open up the translation corridor through the mechanism.

The International Space Station has seen 13 years of utilization of docking and berthing mechanisms. The common ancestor of most of these mechanisms is the APAS, which has roots in the Apollo/Soyuz program. It is the common ancestor of the next generation of low impact docking mechanisms, and the international docking standard. Boeing is leading the development of this docking standard, continuing the tradition of leadership that we have upheld since the first docking.

\section{Acknowledgments}

The authors wish to aknowledge Mike Raftery, who originally inspired this paper, and Kevin Foley and Mark Ortiz for mentorship. Thanks also go out to Michael Brown for providing insight. Additional thanks go to Craig Stanton, VJ Bolton, Elliot Harik, Kirsty Reidy, Carter Reznik, and William J Easton for researching photos of various interface mechanisms. Bob Foster's original and much more detailed Interface Mechanism Topology chart inspired the much simplified version shown in this paper. 


\section{References}

\section{[1] “1-2-3 AND THE MOON - PROJECTS MERCURY, GEMINI, AND APOLLO OF AMERICA'S MANNED SPACE FLIGHT PROGRAM ,” NASA EP-7, 1963.}

[2] Dotts, H.,Nolting,R., Hoyler, W., Havey, J., Carter, T. and Johnson, R., “Operational Characteristics of the Docked Configuration,” Gemini Summary Conference, NASA SP 138, Houston, TX. 1967, pp. 42.

[3] “Manned Space Flight: Projects Mercury and Gemini ” NASA NF- 9/VOl. II. NO. 8, 1967, pp. 9-10.

[4] Orloff, Richard W., Apollo by the Numbers, $2^{\text {nd }}$ ed., Springer-Verlag, New York, 1996, Chaps. 7, 14.

[5] Langley, R., “The Apollo 14 Docking Anomaly,” $7^{\text {th }}$ Aerospace Mechanism Symposium, NASA TM X-58016, Houston, TX. 1972, pp. 197.

[6] Dorland, W., “Dynamic Testing of Docking System Hardware,” $7^{\text {th }}$ Aerospace Mechanism Symposium, NASA TM X-58016, Houston, TX. 1972, pp. 210.

[7] Godwin, Robert (ed.), Rocket and Space Corporation Energia, Apogee Books, Ontario, Canada, 2001, pp. 124.

[8] Yenne, Bill, Encyclopedia of US Spacecraft, Bison Books, London, England, 1985, pp. 173.

[9] Swan, W, “The Apollo -Soyuz Test Project Docking System,” 10 ${ }^{\text {th }}$ Aerospace Mechanism Symposium, NASA TM 33-777, Pasadena, CA., 1976, pp. 32.

[10] Swan, W, “The Apollo -Soyuz Test Project Docking System,” 10 $0^{\text {th }}$ Aerospace Mechanism Symposium, NASA TM 33-777, Pasadena, CA., 1976, pp. 34.

[11] Swan, W, “The Apollo -Soyuz Test Project Docking System,” 10 $0^{\text {th }}$ Aerospace Mechanism Symposium, NASA TM 33-777, Pasadena, CA., 1976, pp. 34.

[12] NASA, “9702655,” NASA Image eXchange URL: http://nix.nasa.gov/ [cited 23 June 2011].

[13] NASA, “ISS017E00941,” DIMS (internal NASA website) [cited 18 June 2009].

[14] NASA, “ISS027-E-036638,”NASA expedition 27 departure photos URL: http://go.nasa.gov/stationportrait [cited 28 June 2011]

[15] NASA, “iss007e13791,” URL: http://spaceflight.nasa.gov/gallery/images/station/crew-7/html/iss007e13791.html [cited 7 July 2011]

[16] RSC Energia, "Purpose and characteristics of the Docking System,” 50 ${ }^{\text {th }}$ Anniversary Yuri Gagarin Flight Exhibition, Moscow, RU., April 2011, pp.2 
[17] RSC Energia, "Purpose and characteristics of the Docking System,” $50^{\text {th }}$ Anniversary Yuri Gagarin Flight Exhibition, Moscow, RU., April 2011, pp.3

[18] RSC Energia, "Purpose and characteristics of the Docking System," 50 $0^{\text {th }}$ Anniversary Yuri Gagarin Flight Exhibition, Moscow, RU., April 2011, pp.3

[19] RSC Energia, "Purpose and characteristics of the Docking System," 50 ${ }^{\text {th }}$ Anniversary Yuri Gagarin Flight Exhibition, Moscow, RU., April 2011, pp.4

[20] RSC Energia, "Purpose and characteristics of the Docking System,” 50 ${ }^{\text {th }}$ Anniversary Yuri Gagarin Flight Exhibition, Moscow, RU., April 2011, pp.4

[21] RSC Energia, "Purpose and characteristics of the Docking System,” 50th Anniversary Yuri Gagarin Flight Exhibition, Moscow, RU., April 2011, pp.8

[22] NASA, “jsc2008e012204,” DIMS (internal NASA website) [cited 15 June 2011].

[23] NASA, “jsc2007e050156,” DIMS (internal NASA website) [cited 15 June 2011].

[24] Stanton, C., “MVC-570F,” Digital PreAssembly Photo Archive, Boeing, Houston, Texas, 2002 (unpublished).

[25] Stanton, C., “762f-PortSSAS,” Digital PreAssembly Photo Archive, Boeing, Houston, Texas, 2002 (unpublished).

[26] NASA, “s127e009988,” DIMS (internal NASA website) [cited 15 June 2011].

[27] NASA, “jsc2000e29475,” DIMS (internal NASA website) [cited 15 June 2011].

[28] Stanton, C., “MVC-856F,” Digital PreAssembly Photo Archive, Boeing, Houston, Texas, 2002 (unpublished).

[29] NASA, “jsc2003e51876,” DIMS (internal NASA website) [cited 15 June 2011].

[30] Stanton, C., “MVC-079X,” Digital PreAssembly Photo Archive, Boeing, Houston, Texas, 2000 (unpublished).

[31] NASA, “jsc2002e02141,” DIMS (internal NASA website) [cited 15 June 2011].

[32] Stanton, C., “821F-NadirUCCASOpenClaw,” Digital PreAssembly Photo Archive, Boeing, Houston, Texas, 2002 (unpublished).

[33] Stanton, C., “188X-PCAS,” Digital PreAssembly Photo Archive, Boeing, Houston, Texas, 2002 (unpublished).

[34] NASA, “jsc2002e29621,” DIMS (internal NASA website) [cited 23 June 2011].

[35] NASA, “jsc2002e17221,” DIMS (internal NASA website) [cited 23 June 2011].

[36] NASA, “s108e5079,” DIMS (internal NASA website) [cited 15 June 2011].

[37] Stanton, C., “058X_EFBM2-HRM3mount,” Digital PreAssembly Photo Archive, Boeing, Houston, Texas, 2003 (unpublished).

[38] Stanton, C., “Overall1,” Digital PreAssembly Photo Archive, Boeing, Houston, Texas, 2008 (unpublished). 
[39] Stanton, C., “EF_SIDE_fromPORT2,” Digital PreAssembly Photo Archive, Boeing, Houston, Texas, 2008 (unpublished).

[40] Stanton, C., “MVC-740F,” Digital PreAssembly Photo Archive, Boeing, Houston, Texas, 2004 (unpublished).

[41] Le, T., Allen, M., “LIDS PDR Mechanical Presentation-final,” Low Impact Docking System (LIDS) Preliminary Design Review (PDR), Houston, Texas, March 31 - April 4, 2008 (unpublished).

[42] Hatfield, S., “NASA Docking System (NDS) Technical Integration Meeting November 17, 2010,” http://dockingstandard.nasa.gov/Meetings/TIM_(Nov-17-2010)/NDS_TIM_presentation.pdf [cited 6 July 2011]. 